\title{
Three-dimensional (3D) liver cell models - A Tool for Bridging the Gap Between Animal Studies and Clinical Trials When Screening Liver Accumulation and Toxicity of Nanobiomaterials
}

\section{Melissa Anne Tutty ( $\square$ tuttym@tcd.ie)}

Trinity College Dublin https://orcid.org/0000-0002-6705-2492

\section{Dania Movia}

Trinity College: The University of Dublin Trinity College

Adriele Prina-Mello

Trinity College: The University of Dublin Trinity College

\section{Research Article}

Keywords: hepatotoxicity, 3D models, nanobiomaterials, liver, nanomedicine

Posted Date: January 19th, 2022

DOI: https://doi.org/10.21203/rs.3.rs-1246403/v1

License: @ (1) This work is licensed under a Creative Commons Attribution 4.0 International License.

Read Full License 


\section{Abstract}

Despite the exciting properties and wide-reaching applications in human health and medicine of nanobiomaterials (NBMs), their translation from bench to bedside is slow, with a predominant issue being liver accumulation and toxicity following systemic administration. In vitro 2D cell-based assays and in vivo testing are the most popular and widely used methods for assessing liver toxicity at preclinical stages, however these fall short in predicting toxicity for NBMs. Focusing on the in vitro and in vivo assessment, the accurate prediction of human-specific hepatotoxicity is still a significant challenge to researchers. This review describes such methods, focusing on the limitations they bring in the assessment of NBM hepatotoxicity, as one of the reasons defining the poor translation for NBMs. We will then present some of the most recent advances towards the development of more biologically relevant in vitro liver methods based on tissue-mimetic 3D cell models and how these could facilitate the translation NBM going forward. Finally, we also discuss the low public acceptance and limited uptake of tissuemimetic 3D models in the pre-clinical assessment, despite the demonstrated technical and ethical advantages.

\section{Introduction}

The many applications of nanobiomaterials (NBMs) have made them extremely beneficial in several fields, most notably medicine. With regard to their medical applications, whilst NBMs offer many new exciting opportunities, their translation is still slow. Up to recent years, the large investments into nanomedicine research, in fact, only yielded relatively small number of products that were successfully translated into the clinic to date [1]. This did shift with the development of two new mRNA vaccines for treating COVID-19 [2], however outside the area of vaccine development progress is still slow. This constitutes one of the main caveats of nanomedicine research field [3]. The high attrition rate of NBMs is partly due to the insufficient and ineffective pre-clinical screening methods that are currently used for testing their toxicity in the body $[4,5]$ The consequence is, the most common reason for withdrawal of nanomedicine products from the clinical market is actually NBM-induced liver injury [6], which is often associated to a considerable liver-specific accumulation [7].

Structurally, the liver is highly vascularised, with its primary function to sequester and remove foreign material from the body, including viruses, bacteria and, indeed, NBMs. Its structure is well adapted for this purpose, with fenestrations in endothelial cells trapping foreign materials in a manner similar to the Enhanced Permeation and Retention (EPR) effect associated with many of the NBM advantages as drug carriers [8].Thus, the anatomy of the liver explains why non-specific liver accumulation and unintended hepatotoxicity are major obstacles in the clinical translation of NBMs. The vast majority of NBMs are in fact administered intravenously, where the liver constitutes the first pass metabolism. For example, Doxil®, the liposomal formulation of doxorubicin currently used in the clinic for many indications including breast and bladder cancer, acute lymphocytic leukemia, and Kaposi's sarcoma, is metabolised mainly via the liver before being eliminated primarily via the biliary system. The consequence is that, whilst Doxilß does reduce some of the negative side effects associated with free doxorubicin, such as 
cumulative and dose dependent cardiotoxicity and neutropenic enterocolitis $[9,10]$, it causes hepatic necrosis [11], among other issues.

From the considerations reported above, it is evident that the liver function is critical in defining both NBMs safety profile and their ADME (absorption, distribution, metabolism, and excretion) once administered to humans. The use of sensitive and human-relevant liver models is therefore vitally important for increasing success with regard to clinical translation of NMBs.

In this context, this review presents the current state of the art in the pre-clinical assessment of NBMs, the limitations of current in vitro and in vivo liver models, and how tissue-mimetic 3D cell culture models can overcome such limitations, assisting in the translation of NBMs to the clinic. Finally, our review offers an insight in the potential reasons determining the limited adoption of these advanced 3D liver models along the preclinical R\&D pipeline.

\section{Current state of the art in the pre-clinical assessment of NBMs}

Since the approval of Doxil ${ }^{\circledR} /$ Caelyx ${ }^{\circledR}$ in 1995 by the Food and Drug Administration (FDA), there has been a yearly increase in the number of biomedical applications for engineered NBMs [12-14]. As for conventional molecular drugs, several in vitro and in vivo pre-clinical tests (listed in Figure 1) are carried out on NBMs prior to starting human trials. Briefly, following pre-screening (including sterility assessment), NBMs enter an assessment cascade covering areas such as physicochemical characterization, in vitro tests (e.g., haematology, cytotoxicity and immunology), and in vivo tests (pharmacokinetics, biodistribution and accumulation).

Focusing on the in vitro and in vivo assessment, the accurate prediction of human-specific hepatotoxicity is still a significant challenge to researchers [15]. Currently, in vitro two-dimensional (2D) cultures and in vivo testing in small mammals are the gold standards for determining acute hepatotoxicity of NBMs prior to clinical trials $[16,17]$. Whilst conventional 2D in vitro cell-based models of the liver are extremely useful as first step in hepatotoxicity testing due to their low cost and ease of use, many limitations reduce their predictive power [18]. Namely the simplicity or the distinct lack of functional cross-talk between the cells forming the model in vitro [19], their poor ability to replicate the in vivo liver-like phenotypic properties and liver physiology [20]. Similarly, in vivo animal models, despite being a key element of the regulatory requirements for both drugs and NBM pre-clinical assessment, are often unable to accurately predict human liver toxicity, due to the fundamental inter-species differences in both organ physiology and NBM uptake, degradation and metabolism [21]. In recent years, scientific efforts focused on the development of new, more "human-relevant" technologies for determining the interactions and potential toxic effects of NMBs in the human liver.

\subsection{Advantages and disadvantages of existing 2D in vitro culture models}

In vitro liver models, in their two-dimensional biological configuration have been long established, nonetheless since the development of 3D biology, several issues have impacted their utilization, as 
summarized in table 1. Given the complexity of the organ and its many roles involved, it is not strange to see that the main limitations for the 2D models reside on the composition and its flat configuration.

As an organ, the human liver is complex and highly vascularized (Figure 2). Connected by the hepatic artery carrying blood from the aorta, and by the portal vein carrying blood from the gastrointestinal tract (GIT), pancreas and spleen, the liver also has a heterogenous cellular composition, incorporating hepatocytes (the primary target of disease and the most abundant liver cell in terms of both volume and quantity), the liver resident macrophages known as Kupffer cells, hepatic stellate cells (HSCs), fibroblasts, immune cells, biliary epithelial cells and adult stem cells [19]. Another essential component of the liver is the cytochrome P450 (CYP450) family of enzymes, vitally important to liver function as they mediate drug metabolism [19]. Bile canaliculi collect bile formed in the liver, which in turn is drained in bile ducts before entering either the gall bladder or duodenum [23]. The liver also exhibits functional zonation, whereby hepatocytes in different zones exhibit different functionality. It is vitally important that liver zonation is considered when undertaking liver modelling, as zonation is disrupted in diseases, particularly ones associated with reactive oxygen species and hypoxia, such as hepatocellular carcinoma (HCC) and non-alcoholic fatty liver disease (NAFLD) [19]. A significant challenge today is the accurate prediction human-specific liver toxicity. It is widely reported that animal models often do not reflect human specific toxicity due to disease adaptations, interspecies variation, and fundamental differences in physiology. Comparably, in vitro models often do not predict toxicity accurately due to significant issues such as nonorgan-specific toxicity, non-linear dose-toxicity, unclear mechanisms, and lack of the key structural and functional characteristics listed above, including lack of liver cell heterogeneity, zonation, formation of secondary structures and in vivo-like cell density [24].

In vitro 2D cell culture models are mainly monocultures formed from human immortalized or transformed cell lines, such as HepG2 [25-29], C3A [30, 31], Huh7 [25, 32, 33] and HepaRG [25, 33, 34] cells. Immortalized hepatic cell lines, normally derived from human hepatocellular carcinoma, have been the "go-to" for researchers for decades for a variety of reasons, including their wide availability from certified cell line sources such as ATCC (i.e. the American Type Culture Collection, which is a non-profit organization that collects, stores, and distributes standard reference cell lines and other research and development materials), or BIOPREDICT (whose most popular product is the HepaRG cell line), relative ease of handling and culture, lack of inter-donor variation [25, 35-37], long-term maintenance in culture with a stable phenotype, and resistance to senescence [38]. For decades, in vitro 2D hepatocyte models have been successfully used to obtain preclinical data on many NBMs, including the liposomal forms of Irinotecan (including studies undertaken in SK-Hep-1 cells) [39] and doxorubicin (HepG2 cells, among others) [40], gold [41-43], SPIONs [44, 45], and carrier NBMs such as Poly(Alkyl Cyanoacrylate, or PACA $[46,47]$ and Poly(Butyl Cyanoacrylate, or PBCA [48].

Despite being widely used as early predictors of NBM liver toxicity in pre-clinical assessment [21, 49], cells lines used in 2D cultures exhibit many disadvantages as presented below. Hepatic cell lines have reduced metabolic capacities, exhibit genomic content and altered phenotypes, upregulated expression of inflammatory mediators, loss of cell polarity and contact inhibition, and, most notably, decrease in 
specific liver function, e.g., reduced CYP450 activity [50-53]. Hepatocytes, cuboidal hepatic epithelial cells that line the sinusoids, are the primary functioning cell type in the liver and make up $80 \%$ of total liver mass. Hepatocyte function ranges from detoxification of toxic materials to basic metabolic functions [54]. They also activate innate immunity, as a defense mechanism against the introduction of external foreign chemicals and substances such as NMBs [55]. It has previously been shown that, to induce an increase in CYP enzyme activity in the HepaRG cell line, the cells must be treated with high concentrations of DMSO, a response which is artificial and counterproductive, as it alters the hepatotoxic responses to NBM exposure and, subsequently, skews data obtained from cytotoxicity assessment [25]. In the HepG2 cell line, the expression of Phase I and II enzymes is also dramatically lower when compared to primary human hepatocytes $(\mathrm{PHH})$, therefore hepatotoxins are not as readily or accurately detected in immortalized HepG2 cell cultures as they would be in vivo.

It is possible to overcome the major caveats of hepatocyte cell lines by using $\mathrm{PHH}$, i.e., cells extracted from human liver biopsies [56-58]. When cultured in monolayers, the PHH metabolic capacity is comparable to in vivo hepatocytes, particularly with regard to albumin secretion, regulation of Phase I and II metabolic pathways, expression of specific liver functional markers, uptake and metabolism, ammonia detoxification and glucose metabolism [59]. This makes PHH the most accurate and physiologically relevant hepatic cell model to date [35]. In spite of this, PHH cultures are not without limitations. Supply of these cells is limited and protocols for extraction are complex. There is also an added issue of inter-donor variation and ability to maintain a stable phenotype, with wild-type characteristics only maintained for a limited time (up to72 hours) in 2D, due to de-differentiation mechanisms induced by the cell growth on flat culture surfaces, reducing the predictive power of the model [60]. Wild-type characteristics are also reduced from handling of the cells in culture, with changes in cell metabolism (e.g., reduction in CYP450 enzyme activity) and senescence observed at low passage numbers $[56,57]$. A further issue is observed with cell seeding density, normally approximately $1 \%$ of physiological density, something which again impacts intercellular signaling and the predictive power of the model $[61,62]$. To avoid these problems, fresh PHH must be extracted from human biopsies regularly. This however incurs in large research costs and dramatically increases the labour and time associated with liver toxicity screening of NBMs based on PHH models. A further way of overcoming issues with the composition of conventional 2D liver cultures, is by forming cultures comprising multiple liver cell types. The liver is an extremely diverse organ, with varied cellular composition containing not only hepatocytes, but also many non-parenchymal cell types (comprising the $20 \%$ of the total liver mass) in the form of Kupffer cells, liver sinusoidal endothelial cells (LSECs), hepatic stellate cells, fibroblasts, biliary epithelial cells, immune cells and adult stem cells [63]. Proper liver function is highly dependent on the interactions between these varying cell types and hepatocytes, therefore by incorporating multiple cell types in a 2D culture it is possible to produce a more physiologically relevant model with tissue characteristics and increased liver functionality $[64,65]$. Recently, many co-culture cell systems have been developed. For example, hepatocyte/macrophage co-cultures have been successfully used to model acute responses to septic liver injury and liver regeneration $[19,66,67]$. Culturing hepatocytes with LSECs, in the presence or absence of collagen, allows LSECs to maintain their phenotype and hepatocytes to increase their in vivo- 
like function also enhanced (e.g., CYP activity) [68]. Similarly, when HepG2 cells are co-cultured with LSECs [69], CYP enzyme induction is enhanced, suggesting that the introduction of endothelial cells influences hepatocyte function in vitro (46). Furthermore, in addition to LSECs enhancing hepatocyte function, similarly hepatocytes support LSECs function [68], influencing LSECs ability to control important repair mechanisms in the liver following injury [70]. Kupffer cell/hepatocyte co-cultures have also been reported, exhibiting responses to a variety of inflammatory stimuli not observed in monocultures [71, 72]. Metabolic functions are also dramatically increased when KCs are cultured with hepatocytes, as demonstrated by Yagi et al. [73]. Various other immune cells have also been co-cultured with hepatocytes [67, 74], such as for example the monocytic THP-1 cell line [75]. Huh7 and THP-1 cocultures expressed pro-inflammatory and stress-related signaling molecules following treatment with troglitazone, a hepatotoxic compound [75]. Furthermore, the co-culture showed increased sensitivity and drug metabolism as compared to Huh7 monocultures [75]. Due to their distinct advantages over hepatic monocultures, co-cultures of liver cells have also been used for the pre-clinical assessment of NBMs. One such study by Esch et al. used a HepG2/C3A co-culture model, incorporated with a Caco-2/HT29-MTX coculture to form a Gl-tract liver system for assessing the uptake, accumulation and toxicity of polystyrene nanoparticles [76]. Despite the many advantages of co-cultures when compared to traditional hepatic monocultures, these models suffer some disadvantages, as summarised in Table 1, and there are fundamental limitations to the number of cell lines that can be cultured together. This issue is associated with others such as determining the appropriate culture environments and conditions, or the lack of essential ECM components, e.g., collagen, which are impacting the cellular phenotype and behaviour and responses to NBMs, as described below.

Another disadvantage of adopting conventional 2D in vitro models is their architecture. Hepatocyte morphology and behaviour is greatly altered due to $2 \mathrm{D}$ cell cultures inability to reproduce the bio-physical cues of human liver connective tissue [77]. Cell behaviour and phenotype is also dramatically affected by the microenvironment across the cell-cell/cell-extracellular matrix (ECM) interactions. In the human hepatic environment, cells have direct contact with each other and the extracellular matrix due to their intrinsic 3D organisation, which is vitally important for cell signalling $[78,79]$. When hepatocytes are cultured in 2D, they lose their 3D interactions and spatial arrangement, and as a consequence their signalling pathways and functions are modified. For the liver to carry out its functions, hepatocytes must exhibit a functional polarised phenotype $[67,80,81]$, with specific transporters localised to both the apical (canalicular) and basolateral (sinusoidal) membrane [67, 80, 81]. Furthermore, in 2D cell cultures the loss of appropriate hepatocyte polarity is thought to be one of the key factors which leads to inaccurate predictions of NBM toxicity in humans. Notwithstanding, unorganised cell proliferation occurs due to the lack of polarisation induced by the two-dimensional environment, a further cause of inaccurate predictions of toxicity $[82,83]$.

Table 1: 2D hepatocyte culture models: advantages and disadvantages. Abbreviations: CYP450, Cytochromes P450; OECD, Organisation for Economic Co-operation and Development. 


\begin{tabular}{|c|c|c|c|}
\hline $\begin{array}{l}\text { 2D } \\
\text { hepatocyte } \\
\text { culture } \\
\text { models }\end{array}$ & Advantages & Disadvantages & Reference \\
\hline \multirow[t]{5}{*}{ Monoculture } & $\begin{array}{l}\text { Current gold standard for drug toxicity and } \\
\text { metabolism studies. }\end{array}$ & $\begin{array}{l}\text { Low predictive value } \\
\text { due to monocellular } \\
\text { composition. }\end{array}$ & \multirow[t]{5}{*}{$\begin{array}{l}12,22-24 \\
27-30,32- \\
33\end{array}$} \\
\hline & \multirow[t]{2}{*}{ Easy and cost effective. } & $\begin{array}{l}\text { Inability to reproduce } \\
\text { liver architecture. }\end{array}$ & \\
\hline & & $\begin{array}{l}\text { Rapid loss of cell } \\
\text { morphology and } \\
\text { polarity. }\end{array}$ & \\
\hline & \multirow[t]{2}{*}{ Small set-up costs. } & $\begin{array}{l}\text { Rapid loss of ability to } \\
\text { metabolize drugs. }\end{array}$ & \\
\hline & & $\begin{array}{l}\text { Decreased albumin } \\
\text { synthesis. }\end{array}$ & \\
\hline \multirow[t]{6}{*}{ Co-culture } & \multirow[t]{2}{*}{$\begin{array}{l}\text { Incorporation of multiple hepatic cell types } \\
\text { means greater in vivo relevance and tissue- } \\
\text { mimetic responses to inflammatory stimuli. }\end{array}$} & $\begin{array}{l}\text { Limitations with the } \\
\text { number of cell types } \\
\text { that can be co- } \\
\text { cultured. }\end{array}$ & \multirow[t]{6}{*}{$\begin{array}{l}37,49-50 \\
51,53-54 \\
56-57\end{array}$} \\
\hline & & $\begin{array}{l}\text { Lack of extracellular } \\
\text { matrix (ECM) } \\
\text { components. }\end{array}$ & \\
\hline & $\begin{array}{l}\text { Hepatocyte-specific morphology/phenotype } \\
\text { is maintained. }\end{array}$ & $\begin{array}{l}\text { High intra-laboratory } \\
\text { variability. }\end{array}$ & \\
\hline & Increased CYP450 enzyme induction/activity & \multirow{2}{*}{$\begin{array}{l}\text { No standard, OECD- } \\
\text { approved co-culture } \\
\text { testing models } \\
\text { established. }\end{array}$} & \\
\hline & Stable albumin production for up to 37 day. & & \\
\hline & Increased phase I and II enzyme expression. & $\begin{array}{l}\text { Optimization is needed } \\
\text { to determine } \\
\text { appropriate culture } \\
\text { conditions. }\end{array}$ & \\
\hline
\end{tabular}

As an organ, , the liver has a heterogenous cellular composition, incorporating hepatocytes (the primary target of disease and the most abundant liver cell in terms of both volume and quantity), the liver resident macrophages known as Kupffer cells, hepatic stellate cells (HSCs), fibroblasts, immune cells, biliary epithelial cells and adult stem cells [19]. Another essential component of the liver is the cytochrome P450 (CYP450) family of enzymes, vitally important to liver function as they mediate drug and NBM metabolism [19]. Each lobe of the liver comprises of approximately one million lobules, around $1 \mathrm{~mm} x$ $2 \mathrm{~mm}$ in size, organised in a hexagonal manner around the central vein. This structure leads to lobule zonation, whereby zone 1 is the periportal zone, closest to the vasculature and most densely supplied with oxygen, nutrients and blood (non-parenchymal cells including hepatic stellate cells and bile duct 
cells are also more abundant in this zone), zone 2 is the transitional region between zone 1 and 3 , and zone 3 , the perivenous zone, is nearest the central vein and less densely supplied with oxygen, blood and essential nutrients $[67,81]$. It is this formation that leads to liver functional zonation, whereby hepatocytes in different zones exhibit different functionality. Conventional in vitro 2D models present significant issues in replicating the human liver, such as lack of cell heterogeneity, zonation, formation of secondary structures and in vivo-like cell density [24].

In detail, in vitro 2D cell culture models are mainly monocultures formed from human immortalized or transformed hepatocyte cell lines, such as HepG2 [25-29], C3A [30, 31], Huh7 [25, 32, 33] and HepaRG [25, $33,34]$ cells. Hepatocytes, cuboidal hepatic epithelial cells that line the sinusoids, are the primary functioning cell type in the liver and make up $80 \%$ of total liver mass. Hepatocyte function ranges from detoxification of toxic materials to basic metabolic functions [54]. They also activate innate immunity, as a defence mechanism against the introduction of external materials such as NMBs [55]. Immortalized hepatocyte cell lines, normally liver epithelial cells derived from human hepatocellular carcinoma, have been the "go-to" for researchers for decades for a variety of reasons, including their wide availability from certified sources such as ATCC or BIOPREDICT, relative ease of handling and culture, lack of inter-donor variation [25, 35-37], long-term maintenance in culture with a stable phenotype, and resistance to senescence [84]. For decades, in vitro 2D hepatocyte models have been successfully used to obtain preclinical data on many NBMs, including the liposomal forms of Irinotecan (including studies undertaken in SK-Hep-1 cells) [39] and doxorubicin (HepG2 cells, among others) [40], gold [41-43], SPIONs $[44,45]$, and carrier NBMs such as Poly(Alkyl Cyanoacrylate, or PACA [46, 47] and Poly(Butyl Cyanoacrylate, or PBCA [48]. Despite being widely used as early predictors of NBM liver toxicity in preclinical assessment [21,49], cells lines used in 2D cultures exhibit many disadvantages as presented below. Hepatic cell lines have reduced metabolic capacities, exhibit genomic content and altered phenotypes, upregulated expression of inflammatory mediators, loss of cell polarity and contact inhibition, and, most notably, decrease in specific liver function such as CYP450 activity [50-53]. It has previously been shown that, to induce an increase in CYP450 enzyme activity in the HepaRG cell line, the cells must be treated with high concentrations of DMSO, a response which is artificial and counterproductive, as it alters the hepatotoxic responses to NBM exposure and, subsequently, skews data obtained from cytotoxicity assessment [25]. In the HepG2 cell line, the expression of Phase I and II enzymes is also dramatically lower when compared to primary human hepatocytes (PHH), therefore hepatotoxins are not as readily or accurately detected in immortalized HepG2 cell cultures as they would be in vivo.

It is possible to overcome the major caveats of hepatocyte cell lines by using $\mathrm{PHH}$, i.e., cells extracted from human liver biopsies [56-58]. When cultured in monolayers, the PHH metabolic capacity is comparable to in vivo hepatocytes, particularly regarding albumin secretion, regulation of Phase I and II metabolic pathways, expression of specific liver functional markers, uptake and metabolism, ammonia detoxification and glucose metabolism [59]. This makes PHH the most accurate and physiologically relevant in vitro hepatic model to date [35]. In spite of this, PHH cultures are not without limitations. Supply of these cells is limited and protocols for extraction are complex. There is also an added issue of 
inter-donor variation and ability to maintain a stable phenotype, with wild-type characteristics only maintained for a limited time (up to 72 hours) in 2D, due to de-differentiation mechanisms induced by the culturing on flat culture surfaces, reducing the predictive power of the model [60]. Wild-type characteristics are also reduced from handling of the cells in culture, with changes in cell metabolism (e.g., reduction in CYP450 enzyme activity) and senescence observed at low passage numbers [56, 57]. A further issue is observed with cell seeding density, which normally equals to approximately $1 \%$ of physiological density, something which again impacts intercellular signalling and the predictive power of the model $[61,62]$. To avoid these problems, fresh PHH must be extracted from human biopsies regularly. This however incurs in large research costs and dramatically increases the labour and time associated with liver toxicity screening of NBMs based on PHH models.

A further way of overcoming issues with the composition of conventional 2D liver cultures, is by forming cultures comprising multiple liver cell types (in vitro co-cultures). As mentioned above, the liver has an extremely heterogeneous composition, containing not only hepatocytes, but also many non-parenchymal cell types (comprising the $20 \%$ of the total liver mass) in the form of Kupffer cells (KCs), liver sinusoidal endothelial cells (LSECs), hepatic stellate cells, fibroblasts, biliary epithelial cells, immune cells and adult stem cells [63]. Proper liver function is highly dependent on the interactions between the nonparenchymal cell types and hepatocytes. By incorporating multiple cell types in a 2D culture, it is possible to produce a more physiologically relevant model with increased tissue-like characteristics and functionality $[64,65]$. Recently, many co-culture cell systems have been developed. For example, hepatocyte/macrophage co-cultures have been successfully used to model acute responses to septic liver injury and liver regeneration $[19,66,67]$. Culturing hepatocytes with LSECs, in the presence or absence of collagen, allows LSECs to maintain their phenotype and hepatocytes to increase their in vivolike function also enhanced (e.g., CYP450 activity) [68]. Similarly, when HepG2 cells are co-cultured with LSECs [69], CYP450 enzyme induction is enhanced, suggesting that the introduction of endothelial cells influences hepatocyte function in vitro (46). Furthermore, in addition to LSECs enhancing hepatocyte function, hepatocytes support LSECs function [68], influencing LSECs ability to control important repair mechanisms in the liver following injury [70]. Kupffer cell/hepatocyte co-cultures have also been reported, exhibiting responses to a variety of inflammatory stimuli not observed in monocultures $[72,85]$. Metabolic functions are also dramatically increased when KCs are cultured with hepatocytes, as demonstrated by Yagi et al. [73].In addition to KCs, various other immune cells have also been co-cultured with hepatocytes [67, 74], such as for example the monocytic THP-1 cell line [75]. Huh7/THP-1 co-cultures expressed pro-inflammatory and stress-related signalling molecules following treatment with troglitazone, a hepatotoxic compound [75]. Furthermore, the co-culture showed increased sensitivity and drug metabolism as compared to Huh7 monocultures [75]. Due to their distinct advantages over hepatic monocultures, co-cultures of liver cells have also been used for the pre-clinical assessment of NBMs. For example, Esch et al. used a HepG2/C3A co-culture model integrated with a Caco-2/HT29-MTX co-culture, to form a Gl-tract liver system for assessing the uptake, accumulation and toxicity of polystyrene nanoparticles [76]. Despite the many advantages of co-cultures when compared to traditional hepatic monocultures, these models suffer some disadvantages such as high variation between cultures, and 
there are fundamental limitations to the number of cell lines that can be cultured together. This issue is associated with determining the appropriate culture environments and conditions for the cell types cocultured, or the lack of essential components of the liver extracellular matrix (ECM) (e.g., collagen) that influence the cellular phenotype and behaviour and responses to NBMs. On this aspect, it should be noted that hepatocyte morphology and behaviour is greatly altered in 2D cell cultures because of the lack of the bio-physical cues characteristic of the human liver connective tissue [77]. Cell behaviour and phenotype is in fact dramatically affected by the microenvironment across the cell-cell/cell- ECM interactions. In the human hepatic environment, cells have direct contact with each other and the ECM due to their tissue 3D organisation. This is vitally important for cell signalling $[78,79]$. When hepatocytes are cultured in 2D, they lose their 3D interactions and spatial arrangement and, consequently, their signalling pathways and functions are altered. For the liver to carry out its functions, hepatocytes must exhibit a functional polarised phenotype [67, 80, 81], with specific transporters localised to either the apical (canalicular) or basolateral (sinusoidal) membrane [67, 80, 81]. The loss of appropriate hepatocyte polarity in 2D cell culture is thought to be one of the key factors that leads to inaccurate predictions of NBM toxicity [16]. The lack of cell polarisation induced by the two-dimensional environment also triggers unorganised cell proliferation, a further cause of inaccurate predictions of toxicity [82, 83].

\subsection{In vivo models for liver toxicity screening: advantages and disadvantages}

In vivo liver toxicity screening tests are vital components of the pre-clinical assessment cascade for NBMs. Animal use in pre-clinical research for in vivo studies do provide a basic overview of the fate of NBMs in organs and provide information on dosing and potential systemic toxicities. Currently, in vivo animal studies for NBMs are most often carried out in porcine or rodent models [86], in accordance with the European Commission Directive 2010/63/EU and the EU legislation on the protection of animals for scientific purposes (which protects live non-human vertebrae and foetal mammals from the last third of their normal development). As just mentioned, rodent models are one the two most commonly used mammal species in in vivo studies. This is due to a variety of reasons including their wide availability, low cost, small sizes, and consequent ease of handling, and fast reproduction rate. Rodents bear close resemblance to humans genetically, biologically and behaviourally, with mice for example sharing almost the same entire set of genes of humans, with less than 10 genes out of the 4,000 studied found in one species but not the other [87]. However, rodent models also show fundamental differences in anatomy, physiology and immune response [88-90].

Pigs are the second most commonly in vivo model used for NBM testing. Many studies have confirmed presence of human-specific cell types in pigs, which cannot be found in rodents [91]. The pig size, coupled with its physical similarities to humans with regard to physiology, anatomy, epigenetics and immunogenetics, make it an ideal models for recapitulating liver-specific diseases ailments such as human hepatocellular carcinoma (HCC), and its co-morbidities, including cirrhosis and non-alcoholic steatohepatitis (NASH) [91]. For example, Andrasina et al. utilised pig models to assess the accumulation and effects of liposomal doxorubicin in liver tissues by radiofrequency ablation and irreversible electroporation [92].In addition to the two models abovementioned, an emerging in vivo model for the pre- 
clinical assessment of NBMs is the zebrafish, a non-mammal model offering new, practical and costeffective opportunities to bridge the gap between in vitro and in vivo studies [93-95]. The recent popularity of this model regarding the study of NBMs is supported by the development of automated, highthroughput readout technologies that can be easily integrated with the zebrafish model [96]. Zebrafish has many characteristics that make it more attractive than conventional in vivo models. Husbandry costs are relatively low compared to rodents, and with the huge financial burdens associated with NBM in vivo studies, this factor is attractive to researchers. Quite possibly the most attractive factor associated with zebrafish is in fact that they can be used without any ethical approval. In accordance with European Commission Directive 2010/63/EU on the protection of animals for scientific purposes, early stage zebrafish are not protected due to their inability to independently feed [97]. Also, young zebrafish, i.e. zebrafish larvae, are optically transparent, enabling high resolution imaging of various events in real time [93]. As with all in vivo models, the question of conservation of characteristics and biological features must be asked prior to implementation as a suitable model for NBM assessment. Zebrafish anatomy and physiology are well described in literature[98, 99], with other physiological parameters and organs of relevance to biodistribution and toxicity of NBMs also extensively studied, including the lymphatic system [100], blood components [101], immune [102] and vascular [103] systems, and the liver [104]. According to these studies, zebrafish share many essential physiological homologies with humans. $76 \%$ of human genes have orthologues in zebrafish, thus being relatively comparable to murine or chicken models, which have $84 \%$ and $80 \%$ respectively [105]. Given the advantages over their rodent counterparts, zebrafish and their larvae are becoming increasingly used as models for the assessment of NBMs, with accumulation, circulation, biodistribution, stability and toxicity all studied in living zebrafish [93] Examples of the use of the zebrafish model in the pre-clinical assessment of NBMs include work by Vibe et al., who assessed the toxicity of thioridazine encapsulated PLGA particles [106], Peng et al. who assessed the release of hydrophobic drugs from cyclodextrin and dextran based nanocarriers [107], and Yan et al. who used zebrafish to study photothermal controlled drug delivery used mesoporous silica nanoparticles [108].

Nevertheless, the choice of the animal model and the awareness of its intrinsic limitations and assumptions are important elements when assessing NBM liver toxicity and accumulation. Despite the deeply rooted assumption among the scientific community that the animal models briefly described above are good predictors of human toxicity, in fact, there is much information which exists to the contrary [109-111]. Only 60\% of drugs entering clinical trials are successful in Phase I (safety) trials [112]. Half of these failures occur due to unanticipated human toxicity, unseen in animal models.

\subsection{Relevance of in vitro models vs. in vivo}

Our provocative question is: if animal studies are truly predictive of human toxicities, why are toxicity rated drug attrition rates in human trials so high? Notably, this is not a concern shared only by the authors, and consensus among several scientists is that in vivo animal models are not as accurate predictors of human responses as we have assumed to date [113, 114]. In 2006, a review of 76 different animal studies found that animal results could be replicated in human randomised trials only in 28 cases ( $37 \%$ of the studies), while animal results were completely contradicted in humans for 14 cases (the 
remaining 34 cases remained untested in humans) [115]. A further review of 221 animal studies showed that only in $50 \%$ of the cases results obtained from animal experiments were in agreement with those generated by human trials. Here, discordance between animals and humans was found to be caused by both experimental bias and the failure of the animal model to adequately mimic human disease state [116]. When concerned with the ability of animal models to accurately predict hepatotoxicity, the issues are similar. A review of 230 animal studies undertaken in 2000 , showed that hepatotoxicity had an extremely poor animal-to-human correlation [117]. This review was not a unique case. The poor prediction of human liver toxicity by animal models has been reported also by other studies $[118,119]$. Several possible reasons have been proposed as to why such discrepancies are seen between animals and humans. The primary reason is linked to intrinsic interspecies differences with regard to both liver physiology and metabolic capacity of animals. Other factors include the heterogeneity of patients' populations, lifestyle, environmental factors, susceptibility, and pharmacogenetic factors that may make patients more sensitive to adverse reactions than their animal experimental counterparts [120]. Notably, the poor predictive value of animal models can, and has in the past, had catastrophic outcomes for patients, either during clinical trials, or months later after a drug was released on market. One example is the case of fialuridine (1-(2-deoxy-2-fluoro-1-D-arabinofuranosyl)-5-iodouracil, or FIAU), a nucleoside analogue that was developed as a potential therapy for viral hepatitis B [121]. Despite not showing any hepatotoxic indications during pre-clinical animal studies undertaken in several animal species (rat, mouse, dog and cynomolgus monkey) [122], 7 of the 15 volunteers enrolled in the Phase I clinical study suffered lactic acidosis and acute liver failure a few weeks into the trial, at doses 100 times lower than those used in the pre-clinical animal studies. 5 of these patients died, and two more only survived after receiving liver transplants $[121,123]$. Over 20 years later, a retrospective study by the US National Academy of Sciences analysed all preclinical toxicity studies of fialuridine and confirmed that in all animal studies undertaken there was no indication that the drug would cause human liver failure. They did however demonstrate that a specific mice model with humanized livers could in fact recapitulate the drug toxicity, again illustrating the importance of being aware of the limitations and assumptions intrinsic within the use of a model[124]. Other examples include the Serazone, a serotonin antagonist and reuptake inhibitor (SARI) drug used to treat depression, and Rezulin, a drug used to help control blood sugar levels in Type I diabetic patients. Both drugs were recalled from the market for inducing severe hepatoxicity, which went unnoticed during the preclinical assessment as a results of the lack of predictability of the animal models used in the studies [125]. Till recently, Abraxane®, an NBMformulation of albumin bound paclitaxel, currently used in the clinic to treat metastatic breast cancer and NSCLC, did not come with any specific guideline for its use in patients with liver dysfunction. This oversight led to one patient suffering grade IV febrile neutropenia, grade III mucositis and grade III nausea/vomiting. As Abraxane is hepatically metabolized via CYP450 enzymes, specifically CYP2C8, a decreased clearance was observed here due to the patients' hepatic dysfunction, yielding severe drug toxicity [126] [127]. These severe side effects further demonstrate the need for bio comparable pre-clinical assessment models that predict adverse outcomes in patients. False positives are another issue in using animal models for liver toxicity screening. False positives can bring to the dismissal of a potentially useful NBM candidate due to liver toxicity in animals, toxicity that does not replicate in humans [111]. 
Indeed, false positives cannot be identified at the moment, cause time and money loss, and have a negative impact on patients' prognosis and quality of life.

To summarise, it is fair to say that animals do not accurately predict human liver metabolism $[113,128]$, and although liver toxicity screening in animals is necessary to meet specific regulatory requirements [129], it is now evident that absorption, distribution, metabolism and excretion (ADME) studies on NBMs are in need for complementary experiments using more human-relevant models [130] [131].

\section{Advanced 3D cell culture models vs conventional pre-clinical methods: advantages and disadvantages}

In recent years, the scientific conversation has switched towards the concept of adopting more humanrelevant preclinical research by focusing on reliability, accuracy and relevance of the testing methods adopted. It is now suggested that animal studies could be either replaced ("1R principle") or at least widely reduced (as part of the "3Rs principle" of replacing, reducing, and refining the use of animals in scientific research) with other more reliable methodologies. Such methodologies, generally referred to as new approach methodologies (NAMs), may act as huge time- and cost-saving technologies in the NBM development pipeline, provided they are validated and their efficacy in preclinical testing is proven. For NBMs, as previously reported by some of the authors [132-138] , 3D in vitro cell-based methodologies are now seen as a vitally important emerging technology in the NBM pre-clinical assessment cascade [12]. In recent years, sophisticated, physiologically relevant 3D liver models have been developed to predict more accurately the hepatotoxicity of NBMs [139]. These models not only recapitulate whole organ physiology, but they are also suitable for repeated exposures and chronic drug testing, an important consideration for NBM pre-clinical assessment [20]. 3D hepatic models have been shown to exhibit similar patterns of NBM transport, adsorption and distribution that are observed in the human liver [140,141], and they also remain both viable and functional for lengthy culture periods, a characteristic which renders them useful for repeated-dose and chronic hepatotoxicity assessment of NMBs [142-144]. Outside of their uses in toxicity screening of NBMs, these newer models also give better insights into the pathogenesis of liver diseases such as NAFLD and steatosis, therefore unlocking the potential for shifting the in vitro NMBs testing from hepatotoxicity testing to simultaneous toxicity and efficacy screening [145]. Key differences between 2D and 3D models, with respect to morphology, in vivo-likeness, and response to materials, among other parameters is described in Table 2. Furthermore, Figure 3 illustrates the varying 2D and 3D in vitro models of the liver, with respect to cell/tissue source, readouts, and endpoints. Table 3 details associated advantages and disadvantages of the varying hepatic models. In the following sections, some of the most common of 3D in vitro models for hepatotoxicity screening are described in detail.

Table 2: Key differences between 2D and 3D culture, regarding morphology, response to materials, in vivo likeness and other key parameters. 


\begin{tabular}{|c|c|c|}
\hline Property & 2D liver cell model & 3D liver cell model \\
\hline $\begin{array}{l}\text { Morphology and } \\
\text { architecture }\end{array}$ & $\begin{array}{l}\text { Sheet-like, flat, stretched cells } \\
\text { grown in monolayers; do not } \\
\text { mimic natural architecture of } \\
\text { liver. }\end{array}$ & $\begin{array}{l}\text { In vivo-like cell shape; high similarities } \\
\text { to in vivo liver architecture. }\end{array}$ \\
\hline Cell proliferation & $\begin{array}{l}\text { Cells proliferate at a higher rate } \\
\text { than in vivo. }\end{array}$ & $\begin{array}{l}\text { Cells proliferate at faster or slower rate } \\
\text { than 2D culture, depending on cell } \\
\text { type/3D system. }\end{array}$ \\
\hline $\begin{array}{l}\text { Protein/gene } \\
\text { expression }\end{array}$ & $\begin{array}{l}\text { Often display different } \\
\text { expression levels to human liver } \\
\text { tissues. }\end{array}$ & $\begin{array}{l}\text { Protein/gene expression levels similar } \\
\text { to those found in human liver tissues. }\end{array}$ \\
\hline $\begin{array}{l}\text { Access to oxygen, } \\
\text { metabolites, nutrients } \\
\text { and signaling } \\
\text { molecules }\end{array}$ & Unlimited access. & $\begin{array}{l}\text { Access is defined by the } 3 \mathrm{D} \\
\text { morphology of the cultures as per in } \\
\text { vivo conditions. }\end{array}$ \\
\hline Cell-cell interactions & $\begin{array}{l}\text { Cannot recapitulate cell-cell } \\
\text { and/or cell-ECM interactions due } \\
\text { to flat morphology. }\end{array}$ & $\begin{array}{l}\text { Appropriate interactions between cell- } \\
\text { cell and cell-ECM are established. }\end{array}$ \\
\hline $\begin{array}{l}\text { Multicellular } \\
\text { composition }\end{array}$ & $\begin{array}{l}\text { Co-cultures can be formed; } \\
\text { number of cell types co-cultured } \\
\text { is limited. }\end{array}$ & $\begin{array}{l}\text { Tissue composition can be fully } \\
\text { replicated (e.g., organoids). }\end{array}$ \\
\hline $\begin{array}{l}\text { Sensitivity to } \\
\text { stimuli/hepatotoxins }\end{array}$ & $\begin{array}{l}\text { Sensitivity is often not } \\
\text { comparable to the in vivo liver } \\
\text { tissue. }\end{array}$ & Better predictors of in vivo responses. \\
\hline Exposure to NBMs & $\begin{array}{l}\text { All cells are equally exposed to } \\
\text { NBMs. }\end{array}$ & $\begin{array}{l}\text { Depending on culture morphology, } \\
\text { NBMs may not penetrate core and } \\
\text { reach all cells, as per in vivo } \\
\text { conditions. }\end{array}$ \\
\hline Reproducibility & $\begin{array}{l}\text { Reproducible high-performance } \\
\text { and simple but highly } \\
\text { reductionist. }\end{array}$ & $\begin{array}{l}\text { Reproducibility depends on method, } \\
\text { can be user-dependent, but it can be } \\
\text { optimized. }\end{array}$ \\
\hline $\begin{array}{l}\text { Cost of maintaining } \\
\text { culture }\end{array}$ & $\begin{array}{l}\text { Cheap, all reagents/materials } \\
\text { commercially available. }\end{array}$ & $\begin{array}{l}\text { Often more expensive, time consuming, } \\
\text { increased batch-to-batch variation. }\end{array}$ \\
\hline
\end{tabular}

Table 3: Advantages and disadvantages of commonly used 3D culture methodologies 


\begin{tabular}{|c|c|c|}
\hline & Advantages & Disadvantages \\
\hline Spheroids/MCTS & $\begin{array}{l}\text { Easy to culture } \\
\text { Mimic in vivo-like cell-cell and cell-ECM } \\
\text { interactions } \\
\text { Scalable and HTS compliant } \\
\text { Easily extracted for further } \\
\text { experimentation }\end{array}$ & $\begin{array}{l}\text { Size variability } \\
\text { Limited diffusion if large } \\
\text { Necrotic core formation } \\
\text { Agglomeration } \\
\text { Take time to form and shown } \\
\text { functionality }\end{array}$ \\
\hline Scaffolds/Hydrogels & $\begin{array}{l}\text { Hydrogels: In vivo-like 3D interactions } \\
\text { Used to study cell } \\
\text { aggressiveness/metastatic potential } \\
\text { Scaffolds: can be combined with } \\
\text { functional tests }\end{array}$ & $\begin{array}{l}\text { Hydrogels: Size/shape variation. } \\
\text { Hard to reproduce } \\
\text { Difficult cell extraction } \\
\text { Scaffolds: Cells can } \\
\text { flatten/adhere to scaffold } \\
\text { Difficult materials can affect } \\
\text { growth }\end{array}$ \\
\hline Organoid/Tumoroid & $\begin{array}{l}\text { In vivo-like architecture } \\
\text { In vivo-like complexity } \\
\text { Patient specific } \\
\text { Replicate in vivo-like cell interactions }\end{array}$ & $\begin{array}{l}\text { Complex to culture } \\
\text { Variation } \\
\text { Less amenable to HTS } \\
\text { Needs much } \\
\text { optimization/validation } \\
\text { May lack certain cell } \\
\text { types/vasculature }\end{array}$ \\
\hline $\begin{array}{l}\text { Liver-On-A- } \\
\text { Chip/Micro } \\
\text { physiological } \\
\text { systems } \\
\text { (MPS) }\end{array}$ & $\begin{array}{l}\text { In vivo-like architecture } \\
\text { In vivo-like chemical/physical } \\
\text { gradients, microenvironment }\end{array}$ & $\begin{array}{l}\text { Flow of medium may disrupt cells } \\
\text { Difficult to adapt to HTS } \\
\text { Lack vasculature }\end{array}$ \\
\hline $\begin{array}{l}\text { Explants/Tumour } \\
\text { Explants }\end{array}$ & $\begin{array}{l}\text { In vivo-like architecture } \\
\text { In vivo-like complexity } \\
\text { Useful in modelling disease }\end{array}$ & $\begin{array}{l}\text { Variation between donors } \\
\text { Difficult to obtain } \\
\text { Complex to culture/expensive to } \\
\text { maintain } \\
\text { Lack long-term viability }\end{array}$ \\
\hline
\end{tabular}

\subsection{Sandwich cultures}

The earliest attempts to recapitulate the complexities of the in vivo liver environment date back to the work of Dunn et al. in 1989, when a hepatocyte sandwich culture model (SCH) was developed using adult rat hepatocytes and a single layer of collagen [146-148]. Nowadays, SCH liver models commonly incorporate two layers of a component of the liver ECM, (e.g., collagen) or a naturally occurring ECM like Matrigel $[149,150]$. Whilst still reductionist due to the lack of the cell-cell interactions that can be observed in the in vivo liver organ, $\mathrm{SCH}$ models do allow the establishment of cell-ECM interactions, an important step up from conventional 2D monocultures. Because of this, a more in vivo-like hepatic environment is promoted, with maintenance of cellular polarity and tissue-like metabolism, formation of gap junctions, and normal levels of key liver specific proteins and substances (e.g., albumin, urea, bile acids) observed [83]. In SCH, formation of intact and functional canalicular networks also occurs and can be maintained over several days in culture $[25,151]$. All of these characteristics make SCH models 
relatively good in predicting human hepatotoxicity, making them useful tools for determining transport, interactions and hepatoxicity of NBMs [25].

\subsection{Scaffold-based cultures}

A popular method employed for 3D culture of hepatocytes is the use of culture matrices as scaffolds for cell growth [67]. Matrigel ${ }^{T M}$ has proven to be very useful in the culture of hepatocyte cell lines for modelling the in vivo liver environment, with a study undertaken by Molina-Jimenez et al. demonstrating that culturing Huh-7 cells in Matrigel ${ }^{\mathrm{TM}}$ as 3D models allows prolonged viability (useful for chronic toxicity studies), hepatocyte polarity, and functional transporters [152]. Matrigel ${ }^{\text {TM }}$ has also been used to culture primary human hepatocytes $(\mathrm{PHH})$ in 3D environments. Bell et al. [84] proved that, when 3D and 2D PHH cultures were formed from the same donors, 3D spheroids were more functionally stable and exhibited a greater sensitivity in detecting hepatotoxins as compared to their 2D counterpart.

Collagen is another natural ECM component commonly used as scaffold for growing hepatocytes in 3D. When cultured with collagen, many hepatocyte cultures exhibit improved urea production, albumin synthesis and CYP450 activity [153]. In recent year, the advancements in material chemistry and material fabrication have led to the design of 3D culture materials that accurately represent the chemistry, geometry and signalling found in the liver ECM. These synthetic substrates have been used for studying the liver toxicity of NBMs, with Kotov et al. forming highly viable HepG2 spheroids with intact junctions using hydrogel scaffolds. Interactions between hepatocytes and CdTe NPs and AuNPs, were studied using this model, and it was observed that the toxic effects of both materials were significantly reduced in $3 \mathrm{D}$ culture when compared to $2 \mathrm{D}$, with phenotypic changes and the tissue-like morphology identified as the major factors implicated in these differences [154].

A further scaffold-based culture technique worth mentioning is 3D bioprinting, which uses manufacturing techniques and computer-assisted technology to form 3D structure from cells and biomaterials, with the overall goal of printing functional tissues and organs [155]. The primary advantage of bioprinting is controlled cell distribution, something which can be an issue when seeding cells onto larger scaffolds. In 2010, the first reported bioprinted liver was formed from HepG2 cells encapsulated in alginate hydrogel and was used for drug metabolism studies [156]. A second notable bio-printed 3D liver model was formed from human-induced pluripotent stem cell (hiPSCs)-derived hepatic progenitor cells, adipose-derived stem cells and human umbilical vein endothelial cells (HUVECs). This model was maintained in culture for over 20 days, and exhibited enhanced hepatic morphological organization, liver-specific gene expression, increased albumin and urea production, and enhanced CYP450 induction[157]. More recently, Nguyen et al. established a novel bio-printed human derived mini-liver formed from HUVECs, hepatic stellate cells (HSCs), and human primary hepatocytes for the in vitro assessment of liver toxicity induced by clinical drugs $[158,159]$. This unique tri-culture 3D model not only allowed for a more relevant assessment of cytotoxicity, but also enabled the measurements of responses specific to each of the cell types.

\subsection{Cell spheroids and multicellular tumour spheroids (MCTS)}


One of the most popular and widely used 3D methodology for culturing hepatocytes to date, is the formation of cellular 3D spheroids [160]. 3D spheroids are small, round clusters of cells which can selfassemble naturally in non-adherent environments [161]. 3D spheroids can closely mimic the cell-cell interactions, as well as biological process occurring in an in vivo scenario. In recent decades they have gained increased attention in both drug discovery and tissue engineering due to the huge array of advantages they have over existing 2D monolayer cultures, and researchers are now looking towards 3D culture and spheroid models increasingly to bridge to gap between reductive 2D monolayer cultures and in vivo animal models. There are a wide variety of methodologies for inducing the formation of cellular spheroids through self-aggregation (presented in Figure 4), each varying in complexity and ease of use.

Common techniques include ultra-low attachment (ULA) surfaces and plates [162], hanging drop techniques [163], the use of rotary/spinner or rocked culture vessels [164], and the use of micro-patterned culturing surfaces [165]. Each of these methods are advantageous for producing hepatic spheroids as they allow hepatic cells to self-aggregate and form microtissues as they would in an in vivo situation, without using scaffolds. Cells form direct cell-cell contacts, produce their own ECM, and maintain viability longer, overcoming some the most commonly seen negative effects of conventional 2D and scaffoldbased 3D models [166]. For example, HepG2 spheroids have been shown to exhibit a strong in vivo-like cellular organisation, enhanced production of albumin and urea, and an upregulation of many genes which play essential roles in lipid metabolism and xenobiotics [167]. Spheroids formed from C3A cells show increased liver-like functionality and are more sensitive to hepatotoxins when compared to C3A 2D cultures [168]. PHH can also be maintained long term in 3D spheroids, with these spheroids remaining viable and functional for over five weeks [169]. This helps to overcome one of the primary limitations of PHH cells i.e., long-term viability. Sustained Phase I and II enzyme expression is also observed in 3D PHH spheroids, along with increased albumin and urea secretion, and expression of various liver-specific markers $[170,171]$. Formation of bile canalicular-like networks is also observed, demonstrating hepatocyte polarisation $[170,171]$. 3D liver spheroids can be grown from hepatocytes on their own (as in the examples above) or from hepatocytes in co-culture with other liver cell types (multicellular liver spheroids). Multicellular spheroids, formed from immortalized hepatic cell lines or $\mathrm{PHH}$ and other nonparenchymal liver cells, including liver sinusoidal endothelial cells (LSECs), Kupffer cells (KCs), or hepatic stellate cells (HSCs), provide an in vitro model with a structure and function which closely mimics the human liver [172]. For example, hepatocyte-HSC co-culture spheroids exhibit the same liver-specific morphological and structural properties as it is observed in monocultures. Additionally, they exhibit a $30 \%$ increase in albumin secretion, maintained up to two months in culture, and enhanced CYP450 enzyme expression and activity $[161,173]$. Co-culture models of KCs and LSECs remain viable for up to 35 days and exhibit enhanced expression of transporters and sensitivity to hepatotoxins [170], making them useful models for liver toxicity screening.

One useful application of hepatic spheroids is in fact in the pre-clinical assessment of NBMs. They can provide an understanding of the NBM toxicity in the context of absorption and penetration within the liver tissue. Also, hepatic spheroids have a longer lifespan, rendering them useful for repeated dose or chronic toxicity testing, a major advantage over traditional in vivo studies $[162,174]$. Hepatic spheroids have 
proved useful in the pre-clinical assessment of many NBMs $[154,175,176]$, and are often used to study conditions such as drug-induced liver injury (DILI) [169], which cannot be accurately predicted using animal models. Hepatic multicellular tumour spheroids (MCTS), i.e. 3D spheroids formed from hepatocarcinoma cell lines, have successfully used for assessing NBM cytotoxicity and for predicting in vivo anti-tumour activity, as detailed by Mikhail and co-workers. Here, a MCTS model was treated with docetaxel (DTX) loaded micelles, showing that spheroids were significantly more resistant to treatment in comparison to the corresponding 2D monocultures and responded in an in vivo-like manner, demonstrating that MCTS are viable and in vivo-like platforms for evaluating the impact of NBMs, in conditions which closely mimic in vivo tumour microenvironment [177].

\subsection{Liver organoids}

In recent years liver organoids have emerged as a highly relevant and useful alternative in vitro model with excellent potential for disease modelling and drug and NBM screening. This model resulted to be advantageous over conventional in vitro models due to their long-term genetic stability, in vivo like organization and their ability to maintain the cellular crosstalk and behaviour of their primary cell counterparts $[178,179]$. Formed from induced pluripotent stem cells (iPSCs), embryonic stems cells, hepatoblasts or organ-specific adult tissue-derived cells (both healthy and diseased), which have the ability to self-assemble and differentiate, these functional 3D hepatic models serve as useful platforms to address a wide variety of research questions, ranging from hepatic development and regeneration, to metabolism and detoxification, and are also extremely representative model diseases of the liver including NASH and NAFLD [179], acting as an excellent resource for studying the human liver in ways that were previously very difficult. Up until recently, 2D hepatocyte cultures were the first in line with regard to drug and NBM metabolism and toxicity screening. However, do to impaired CYP450 activity, they are unstable and lacking functionality. As detailed by Mun et al., hepatic organoids help overcome these limitations as they have been shown to express Phase I drug metabolism and Phase II detoxification enzymes. In this study, following treatment with omeprazole, CYP1A2 and 3A4 induction was observed in hepatic organoids. Additionally, when treated with the hepatotoxic drugs troglitazone and APAP in conjunction with 2D hepatocyte cultures, the organoids exhibited a markedly higher sensitivity than the 2D cultures [180]. These organoids have been extensively investigated as most recently reported in the comprehensive reviews published by Prior and co-workers [179] [180].

Liver organoid models have been applied to the pre-clinical assessment of the biocompatibility and toxicity of NBMs. For example, Kermanizadeh et al. have assessed the hepatotoxicity of AgNPs using hepatic organoids formed from human primary hepatocytes and NPCs. Here, following organoid characterisation, various parameters, including cytotoxicity and genotoxicity, were assessed following NBM treatment [30]. Hepatic organoids have also been applied to the assessment of complex NBM systems, such as the liposomal DNA origami nanosystem (LSTDO) designed by Palazzolo et al. Doxorubicin was loaded inside the LSTDO, and the system tested in both murine hepatic organoids and in vivo xenograft mice, to determine if such a system would be clinically relevant and aids to improvements on the current cancer treatment. Before beginning the in vivo mouse study, 
biocompatibility was ensured by undertaking toxicity screening using the mouse-derived hepatic organoid model, and it was found that the DNA origami internalization into liposomes negated many of the toxic effects of free DNA origami. Furthermore, it was found that the combined properties of the liposomes and DNA origami facilitated the introduction of doxorubicin in a biocompatible manner, improving drug accumulation at the tumour site, and inhibiting tumour growth in the mice [181].

To the best of our knowledge, the wider use of human derived liver organoids in the preclinical assessment of NBMs is still in its infancy, and little information is currently available on their wider applications for screening next-generation NBMs such as liposomes or SPIONs. Despite the huge interest in liver organoids, there are not exempted from shortfall and limitations, such as the accurate recapitulation of the in vivo ECM. Others are the lack of a native microenvironment, a problem which can hinder the study of stem cell and niche interactions; the lack of necessary growth factors and an inability to accurately model the immune response. One possible solution for overcoming some of these issues could be the application of microfluidics to these models, as we are discussing in the following sections.

\subsection{Ex vivo models of the liver: precision cut tissue slices, whole organ explants and tumour explants}

Ex vivo models include precision cut tissue slices (PCTS), whole organ explants and tumour tissue explants. PCTS are generated by cutting slices of a defined thickness from viable liver tissue, often from rodent tissues but in more recent years also from humans [182], with human tissues for PCTS normally obtained from either explanted tissue, partial hepatectomies, tissue unsuitable for transplant, or offcuts from surgery (i.e. diseased liver obtained from cirrhotic or fibrotic patients)[183]. PCT allow retaining tissue structure, morphology and intracellular polarization for up to five days in culture (15 with optimal culture conditions) [184]. This offers a model characterized by strong in vivo relevance and with great relevance for hepatotoxicity screening. In PCTS, the multi-cellular histoarchitecture of the liver environment is maintained, including liver-infiltrating immune cells [185]. PCTS are also useful due to their reproducibility, ease of maintenance, and low cost, making them valuable tools for assessing many elements of various liver diseases [183]. A number of studies have demonstrated that interactions between specific liver cell subtypes are conserved in PCTS, with Olinga et al. illustrating the interactions between Kupffer cells and hepatocytes. [186]. Moreover, the ECM, which is notably absent in hepatocyte 2D cultures and 3D spheroids, is also conserved, ensuring the regulation of important cellular functions by associated hormones, cytokines and growth factors $[187,188]$.

To date, a number of studies have used PCTS for NBMs assessment, as PCTS make it possible to test NBM in a multicellular context in a single in vitro model, once which closely resembles in vivo conditions. Dragoni et al. investigated the uptake and toxicity of AuNPs using rat liver PCTS. Here, uptake of AuNPs was observed in hepatocytes, Kupffer and endothelial cells in the liver slices. A more recent publication from Bartucci et al. used, for the first time, human liver slices to investigate ex vivo the NBM behaviour in the liver and to investigate the basic mechanisms of NBM interactions in real time. The findings determined that, as per in vivo conditions, Kupffer cells accumulated large amounts of NBMs, which interestingly move within the tissue slices to the borders [189].However, despite the usefulness of PCTS in 
the pre-screening of NBMs, it is worth noting that they cannot be used in a high throughput manner and rapidly loose functionality days into culture, factors which somewhat limits their usefulness and application [190].

A further advanced ex vivo model, whole organ explants, which can either be suspended in culture medium or embedded in ECM substrates, offer in vivo-like 3D architecture and gene expression which can be maintained up to five days in culture [191]. Whilst conventional studies of the mechanisms and progression of liver disease normally focus on a selection of liver cells and cell culture techniques, whole organ explants offer great advantages over these methods as they not only contain all liver cell populations, but also have their 3D architecture intact. In the past, whole organ explants have been used for studying alcohol-induced liver injury, by exposing them to ethanol [192]. In a similar manner, tumour liver explants can also be used as 3D ex vivo models of HCC, for drug efficacy screening [193, 194]. A further study by Piera et al. used liver explants from mice inoculated with a HCC cell line, Hepa1/A1, to study the antineoplastic potential of a well-known antioxidant, Citozym [195]._Despite their many uses and in vivo relevance, whole organ and tumour liver explants do have many limitations associated with them. These includes the lack of reproducibility due to inter-donor heterogeneity, lack of viability long term, and issues with availability, rendering them unfeasible models for HTP assays or any chronic toxicity screening [196]. Whilst they are becoming more widely used for modelling disease and for assessing material toxicity, information regards their applicability to NBM pre-clinical assessment is still scarce.

\subsection{Liver-on-chip and microphysiological systems (MPS)}

One example of liver-on-chip for in vitro screening of hepatotoxins is the 3D HepaTox Chip, designed by Toh et al. in 2009 [197]. It is considered as one of the first demonstrations of microfluidic system that could accurately prediction hepatoxicity, data obtained using this model strongly correlates to in vivo data [197], with hepatocytes maintaining a comparable, if not higher, concentration of phase I and II metabolic functions when compared to monocultures. After 24 hours in culture, basal CYP 1A1 and CYP1A2 levels were approx. 3-fold higher in the HepaTox Chip than in multiwell plates [197]. Other research undertaken using C3A cells and HepaTox Chip showed that multiple liver specific functions including albumin synthesis, gluconeogenesis and ureagenesis were at almost comparable levels to those of primary hepatocytes [198]. Some of the most recent 3D culture advances have been in this area, and many integrated liver-on-chip microsystems have been developed which reproduce many key structural, functional, biochemical and mechanical features of living organs, in a single, small device [145]. Liver-on-a-chip systems bear a close resemblance to the liver sinusoid, with endothelium separating hepatocytes and a constant flow of nutrients, metabolites and oxygen through the microfluidic channel. Microfluidic "liver-on-chip" models can overcome this issue, allowing for a continuous perfusion of culture medium, nutrients and/or test compounds to the cell monolayer or cell spheroids, generally improving not only viability and lifespan of the cells in question, but also their metabolic performance $[199,200]$. 
Other more sophisticated systems also exist, including hollow-fibre bioreactors. Here, cells are seeded on complex scaffold with a consistent flow of culture medium present. One such study from Gerlach et al. successfully cultured human primary hepatocytes and non-parenchymal cells (NPCs) using this method, with viability maintained for at least three weeks, with vascular cavities and bile-duct like, canalicular structures also visible [201]. Over the years, several microfluidic systems have been developed, with many of them now commercially available through companies like Mimetas, TissUse and Emulate, among others, therefore facilitating their use as emerging hepatic technologies. These novel microfluidic hepatic models have also acted as a new platform for testing NBMs.

As NBMs exhibit differential behaviour depending on whether they are under static or flow conditions, the development of dynamic microfluidic systems are vitally important for accurate determination of NBM toxicity [202]. A primary disadvantage of conventional in vitro models is the issue of providing a constant flow of culture medium/nutrients to the cells, and the artificial way medium is renewed sequentially, to either keep cells alive and healthy, or to introduce NBMs for toxicity screening. The addition of NBMs and culture medium results in non-steady state conditions because of diminishing substrate concentrations, accumulation of product, and other issues such as evaporation over time [203]. Notable examples of this include a study from Li et al. who developed a 3D microfluidic hepatocyte chip for assessing the hepatotoxicity of superparamagnetic iron oxide nanoparticles (SPIONs). Here, primary rat hepatocytes were used to fabricate a three-layer chip which tested SPIONs in short- and long-term culture [204]. A further study from Liu et al. incorporated electrospun fibres in a PDMS microfluidic chip and used this device to culture primary hepatocytes for assessing AgNP hepatotoxicity. Here, hepatocyte behaviour was studied, and it was determined, that by using this platform under optimised flow conditions, specific hepatocyte functions including polarity and biliary excretion were restored and maintained for up to 15 days. Hepatocytes under a $10 \mu \mathrm{L} / \mathrm{min}$ flow rate also produced sensitive and consistent toxicity responses to AgNP, demonstrating the applicability of this model for the in vitro toxicity screening of NBMs [205].

It is also possible for liver-on-a-chip models to be incorporated into a microphysiological system (MPS), where several organs-on-chips are interconnected [206]. Initially, MPS have been developed as a mean for increasing efficiency, speed and safety in the pre-clinical development and assessment of pharmaceuticals. This was born from the inability of 2D monocultures of immortalized cell lines or in vivo animal studies to sufficiently recapitulate the dynamic of drug-drug, drug-organ and drug-organorgan interaction in humans. More recently, MPS have been utilized to assess efficacy and safety of NBMs in one single, compact in vitro platform, ultimately advancing the translation of these materials [207]. A recent example of MPS where a GI/liver MPS incorporating co-cultures of Caco2/HT29-MTX and HepG2/C3A liver cells has been successfully applied for investigating first pass metabolism of high doses of polystyrene nanoparticles intended for daily human consumption [208]. This study determined that despite the nanoparticle permeability across the GI barrier was low, the single nanoparticles and small clusters which did in fact pass through the GI barrier induced aspartate aminotransferase (AST) release in the liver cells, indicating potential liver injury. Overall, sophisticated MPS are a unique model that can offer fundamental understanding of NBM effects in the human body [209]. 
Despite the wide array of benefits associated with microfluidic models, due to their complex nature they are not without fault. Disaggregation and loss of cells can occur due to the constant perfusion of the culture medium, especially as cells proliferate and reach confluence. Additionally, the effect that sheer stress has on the cultured cells is not fully understood yet [210]. Finally, the large set up and maintenance costs associated with them models and systems, as well as the large cell densities needed to build the models and the associated issues with their handling and analysis are impacting or limiting the uptake of such complex platform technologies.

\section{Advanced 3D cell culture models in preclinical testing: Translational approaches}

This review thus far has presented the many challenges associated with the screening of NBM-induced hepatotoxicity, in depth, ranging from the advantages and disadvantages associated with conventional in vitro and in vivo models, to the new, emerging $3 \mathrm{D}$ technologies in this field. The latter offer many exciting opportunities for evaluating the accumulation and hepatotoxicity of NMBs in a human-relevant manner. Not only do they have the potential to closely mimic the specific phenotype and organotypic function of the human liver tissue, allowing a more sensitive and physiologically-relevant assessment of the fate and toxicity of NBMs [154]. They also yield more cost and time effective studies of NBMs and have greatly benefited the wider scientific community since their conceptualisation and development. The effects triggered by NBMs in 3D models differs greatly for those observed in conventional 2D monolayer cultures, a phenomenon demonstrated in many studies, including one by Eljie et al. who used HepG2 cell spheroids for assessing the genotoxic and cytotoxic potential of three nanoparticles, namely silver, zinc oxide and titanium oxide [175]3D cultures are indeed a much more faithful representations of the in vivo hepatic environment when compared to conventional monocultures. Furthermore, these models represent an attractive opportunity in keeping with the 3Rs concept, as a more human-relevant platform or as replacements to animal studies. This is particularly relevant in the EU, as the European Medicines Agency (EMA) has supported the implementation of both Directive 2010/63/EU [97], legislation on the protection of animals used for scientific purpose, as well as the 3Rs principle of replacement, reduction and refinement, which they have supported from as early as 1986 (Directive 86/609/EEC) [211], when the first legislation for the protection of animals used in scientific research was implemented. Since then, the EMA have not only promoted the ethical use of animals in pre-clinical testing, but now also encourage nonanimal alternatives and strive to improve the welfare of animals when their use can't be avoided. Directive 2010/63/EU aims to harmonize all animal research legislation through all EU member states, ensuring that high standards of both scientific research and animal welfare are met. In the EU to date, animals can only be used in research where convincing justification has been put forward, whereby the research benefits outweigh an animal suffering, and when the specific objectives of a study cannot be met using non-animal alternatives. At all times these strict ethical guidelines must be met.

To date, a variety of advanced liver technologies have been successfully utilised in many areas, ranging from the functional, geometric liver models generated by the Max Planck Institute for studying the varying stages of liver disease [212], to the work undertaken by Organovo, a San Diego based company who have developed a bioprinting process whereby liver cells are extracted from donor organs and turned into 
printable bio-ink $[159,213]$ to build up small sections of liver tissue. Today, this technology is expected to revolutionise the drug safety screening. In December 2017, for example, the FDA granted orphan drug status to Organova for their treatment of alpha-1 antitrypsin (A1AT) deficiency based on tests carried out using their 3D bioprinted liver tissues.

It is safe to say that the revolution of bioengineering complex liver models is well and truly underway. In recent years we have witnessed superior manufacturing and machine development, improved bioinks and a huge influx of published literature which leverages $3 D$ bioprinting as a one of the primary tools which will revolutionise and strengthen regenerative medicine in the coming years. In the US and founded in 2009, the Wyss Institute for Biologically Inspired Engineering at Harvard University is at the forefront of liver-on-chip research. Formed from the understanding that research 'can't change the work unless it never leaves the lab' [214], the Wyss Institute is a cross-disciplinary biomedical engineering research institute focusing on the development of bio-printed material and devices which can be utilised for applications in manufacturing, energy, robotics, architecture and the healthcare sector. The WYSS institute are also at the forefront of organ on chip technology. In the last decade the WYSS Institute have developed and validated more than ten different organ-on-a-chip models, including the liver, and have also engineered a device which automates chip processes and can link the different organ-on-a-chip models fluidically to form 'body-on-a-chip' models which recapitulate whole body physiology while allowing high-resolution analysis and imaging. The WYSS institute have successfully translated these innovations into commercial technologies, and have leveraged financial support from the FDA, NIH, and the Defence Advanced Research Projects Agency (DARPA). Their technologies have also been refined and validated for the clinical and market need, and now test existing medicines and model various diseases using their 'on-chip' models in partnership/conjunction with various major pharmaceutical companies [214].

The move towards more complex liver models is further reflected in one of the latest EU Horizon 2020 funded projects, ORGANTRANS (controlled ORGANoids transplantation as enabler for regenerative medicine TRANSlation). This project aims to develop a 3D printed liver tissue platform to potentially replace liver transplantation for patients with end-stage liver failure, using bio fabrication knowledge from the bioengineering company regenHU. Coordinated by CSEM, the Swiss research and development centre, the ORGANTRANS project consortium, made up of two transplantation centres and eight partners, to generate novel techniques which can not only be applied to hepatic systems but also other organ systems in regenerative medicine and will cover the complete cycle of development from cell sourcing and tissue bioengineering to clinical trials. Another Horizon 2020 funded project, PATROLS (Physiologically Anchored Tools for Realistic nanomaterial hazard aSsessment) also aims to develop more physiologically relevant in vitro 3D GIT, lung and liver models which may be incorporated into the pre-clinical testing of ENMs. This project was launched in January 2018 and will run for 3.5 years and involve 24 partners across the USA, Europe and Asia. To date, the PATROLS project have delivered outcomes which include the application of 3D HepG2 spheroids to the genotoxicity screening of ENMs $[215,216]$, the development of complex co-culture hepatic 3D models for ENM toxicity screening [217], various in vitro lung systems for assessing nanomaterial-induced lung toxicity and inflammation [218-220], and a GIT model [221].

Page 23/48 
With regard to outside the EU and US, in the last year scientists at the Human Genome and Stem Cell Centre (HUG-CELL) at the University of Sao Paulo (USP), funded by the Sao Paulo Research Foundation, have succeeded in forming hepatic organoids from induced pluripotent stem cells (iPS) bioink, which are not only capable of performing liver-specific function such as bile secretion and storage of vitamins and proteins, but also have prolonged tissue survival over a long culture period in vitro. Using their method, hepatic tissue can be created in the lab in 90 days, and it is expected that this technology will, in the coming decades, become a feasible alternative to organ transplantation [222].

In China, a collaboration between the Department of Liver Surgery at Peking Union Medical College (PUMC) Hospital, the Chinese Academy of Medical Sciences, Beijing, and the Department of Mechanical Engineering, Beijing has led to the development of 3D bioprinted hepatorganoids (3DP-HOs), constructed from HepaRG cells and bioink, using 3D printing procedures in order to overcome the shortage of organ donors, a critical issue for patients with end-stage organ failure. Following implantation into mice models of liver injury, the hepatorganoids in this study matured to display increased synthesis of liver-specific proteins and drug-metabolism activity. The formation of functional vascular systems was also observed, enhancing liver function and material transport further. Perhaps the most important finding what the fact the 3DP-HOs improved survival of the liver injury mouse model. This study has further emphasised the potential value of 3D bioprinting in the regenerative and transplant medicine field, with this technology allowing the implantation of artificial organs into the human body, ones that demonstrate normal physiological function in vivo, and ones which may offer novel solutions to treat disease [223]. Also, in China, scientists at the Hanghou University of Science and Technology have developed a technology called Regenovo, a medical grade 3D bioprinter which to date has been used to produce numerous gelatinous and semi-transparent 3D bioprinted kidneys, ear cartilage and micro livers using scaffolds containing live cells, inorganic materials and hydrogels, and medical grade polymers. This printer using an $80 \mu \mathrm{M}$ printer nozzle, which allows the printing of cells up to five times greater than normal cells, with cells remaining viable for up to four months. Cell damage rates are also extremely low, with approximately $90 \%$ of cells surviving the printing process [224].

Similarly, in Japan, researchers from the University of Tokyo have also successfully bioprinted scaffoldfree 3D micro livers which were capable of stably maintaining metabolism of essential materials like glucose, lipids and drugs, as well as production and secreting bile. Using the Regenova technology (not to be confused with Chinese Regenovo) designed by Cyfuse, a Japanese biomedical company, liver bioprinting was undertaken using the Kenzan method. Developed by Professor Koich Nakayama and Cyfuse Biomedical, the Kenzan method is a scaffold-free method which eliminates the need for supporting scaffolds. By removing the scaffolds, cells functionality could be extended as cells were not dependant on any outside support from a scaffold. The liver structures formed using this method exhibited self-organization, ECM production and a wide away of other metabolic functions that could be maintained over long periods. They anticipate that this unique liver model may become widely used in drug discovery studies, and may assist with the identification of new drug targets, safety studies and pharmacokinetics [225]. 
With these promising advances observed worldwide, regulators are moving into the 3D space in order to accelerate drug discovery. In the US, the NIH have a program which develops bioprinted tissues, in collaboration with Organovo, with the goal of speeding up drug screening. NCATS, or the National Centre for Advancing Translational Sciences, acknowledge that the current methods for developing and delivering drugs to patients takes decades, incurs huge costs up to billions, and approximately $95 \%$ of the time are unsuccessful, and therefore have launched a 3D tissue bioprinting program, combining living cells and scaffolds to test materials in platforms that closely mimic human tissues and organs in order to better predict human responses to potential therapies [226]. NCATS have also developed 'on-chip' technology with their Tissue Chip for Drug Screening initiative [227]. It can been noted however that despite the work done in NCATS and other institutions, many challenges still remains to successfully construct various 3D bioprinting generated functional organs and actually apply them to pre-clinical assessment and regenerative medicine, particularly with regard to obtaining FDA approval, and despite having moderate success with producing candidates for rare diseases that have been picked up by pharma and which have reached clinical trials, the only FDA approval they have achieved was in 2015 when they showed that a drug previously used to prevent organ rejection could be used to treat a rare lung disease, lymphangioleiomyomatosis (LAM) . Dr Chris Austin, a neurologist at the NIH and director of NCATS, acknowledges that many roadblocks exist to drug development, and include the fact that advanced methodologies for pre-clinical assessment such as 'on-chip' technology, induced pluripotent stem cells and 3D bioprinted organs have yet to be widely accepted and adopted by drug companies as in vitro alternatives to conventional animal tests, and that the pharma industry is 'conservative' and slow to embrace new technologies [228]. A key issue can also be seen in the failure of the FDA to lay out specific pathways for accepting these new technologies in lieu of animal experimentation [228]. One success can be observed in the orphan drug designation Organovo received in 2017 from the FDA for their 3D bioprinted liver tissues used in the treatment of alpha-1 antitrypsin (A1AT) deficiency [229]. Following this designation however, another key issue became apparent, the huge costs incurred from 3D bioprinting, and despite Organovo anticipating that by the end of 2019 they would have two liver tissues on track for 2020, increased costs and extended timelines have pushed human testing back to 2021.

Despite the future looking promising from both a technical and scientific perspective with regard to these advanced technologies, many regulatory hurdles surrounding them exist. One such hurdle is the classification of tissues and organs bioprinted using 3D methods. Should a 3D printed liver be classed as an organ or a product? And with regard to regulation, is it a medical device? These are questions that regulators must answer in order to advance this area, and decisions need to be made as to whether new regulations should be developed for bioprinting or should they be covered under existing frameworks. Some commentators in the EU and US have also raised the issue of morality surrounding bioprinting tissues and organs and have questioned whether bioprinted materials should fall under patent protection, reminiscent of the Dolly the sheep case. A study at Bournemouth University and funded by the EC published in April 2020 has assisted these issues and provides legal guidance on the regulatory and intellectual property issues surrounding 3D bioprinting technology, and it is acknowledged today that if EU regulatory class bioprinted products as medical devices, some degree of clarity will exist legally as 
guidelines for medical devices have been in place for decades. In the US, the FDA have issued guidelines on 3D bioprinted medical devices themselves, but not on the specifics of their printing. These guidelines are also not legally binding, meaning ambiguity still exists. These is no doubt that the era of 3D bioprinting will significantly advance modern medicine and the lives of countless patients, however for this to happen, policymakers and regulators must ensure that the correct guidelines and regulatory are in place. However, optimisation must be undertaken before they can be implemented widely as pre-clinical assessment tools for NBMs or other materials (e.g., active pharmaceutical ingredients, health technology or theranostics). To date, no standardised or validated 3D model, either a healthy or diseased state, is approved for preclinical screening of NBM liver toxicity at the regulatory level. Hence, the need for alternative models which can be successfully integrated into the NBM screening pipeline is still unmet.

\section{Conclusions}

In conclusion, the vast array of applications for NBMs have made them extremely beneficial in many fields, and they offer many exciting opportunities in medicine, however despite their advantages their translation to the clinic is overwhelmingly slow and despite great investment, in recent decades only a small number of NBM-based products have been translated into viable medical products. [1]. This lack of translation and indeed high attrition rate is in large part due to inappropriate pre-clinical assessment screening methodologies for testing NBMs toxicity, in particular hepatotoxicity. Whilst conventional 2D in vitro monoculture liver models formed from immortalized or transformed cell lines are long established and widely used due to their distinct advantages including lack of donor variation and resistance to senescence, they have reduced metabolic capacities, altered phenotypes, loss of polarity and contact inhibition and a decrease in liver specific function, among other key failings. In vitro models also fall short in predicting toxicity due to issues such as non-organ-specific toxicity, non-linear dose-toxicity, unclear mechanisms, lack of the key structural and functional characteristics previously detailed and which include hepatic zonation, secondary structure formation, and in vivo-tike cell heterogenicity, and hepatocyte polarity, a key factor which leads to inaccurate prediction of NBM toxicity. Whilst some of these issues may be overcome by using $\mathrm{PHH}$, supply of these cells is limited, inter-donor variation and difficulties in maintaining a stable phenotype due to the $2 \mathrm{D}$ culture environment could be other limiting factors [56-60]. A further way for overcoming key hurdles seen with conventional liver cultures, the culture of multiple liver cell types in co-culture models, does allow some advantages over monoculture modes however as detailed in Table 1, however there are limitations on how many cell lines can be cultured together, great variability is observed and there is a distinct lack of ECM components, all of which impact the cells response to NBMs.

On the other arm of in vivo liver toxicity screening methods are animal models, used to provide a basic overview of the fate of NBMs in organs, and provide information on dosing and potential toxicities. In vivo animal models for the screening NBMs are most often undertaken in porcine or rodent models [86], in accordance with the European Commission Directive 2010/63/EU, and today they are regarded as an essential component in the translation of NBMs from lab to clinic. Nevertheless, in a similar manner to in vitro methods, they are not without the own intrinsic limitations. Whilst rodent models incur low costs, are 
small and easy to handle, reproduce fast and bear close resemblance to humans in terms of genetics and biology, there are fundamental differences between human and rodent anatomy, physiology and immune response [87-90]. Whilst porcine models are useful in the pre-clinical assessment of NBMs due to the presence of human-specific cell types which cannot be found in rodents [91], and their more similar physical size and similarities to humans with regard to physiology, anatomy, epigenetics and immunogenetics, porcine models are costly, and one must wonder why if animal studies are so predictive of human toxicity are drug and NBM attrition rates still so high? Today consensus among many scientists is that in vivo animal models are not as accurate at predicting human response as we may have previously assumed $[113,114]$, particularly regarding hepatotoxicity [117-119]. These issues, coupled with the need for newer, more bio-comparable assessment models for predicting patient outcomes are what has, in recent years, driven the development of 3D methodologies which may act as a bridge between conventional 2D and in vivo.

These 3D methodologies are now seen as vitally important emerging technologies in the NBM pre-clinical assessment cascade [12], and in recent years, sophisticated, physiologically relevant 3D liver models that can more accurately predict the hepatotoxicity of NBMs have been developed [139]. These models not only recapitulate whole organ physiology, but are also fit for repeated exposures and chronic drug testing, an important consideration with regard to NBM pre-clinical assessment [20]. These 3D cell culture models, namely cell spheroids and MCTS, scaffold and sandwich cultures, organoids, ex vivo models, and more complex models such as MPS, 'on-chip' and bioprinting technologies may act as huge time and cost-savers in the NBM development pipeline, overcoming some of the key challenges faced in NBM assessment, provided the appropriate validation is undertaken and their efficacy in preclinical testing is proven.

From the work undertaken at the Max Planck Institute [212], to Organovo's bioprinting [143, 203], advanced liver models are set to revolutionise drug and NBM safety screening, with funding agencies and regulators now paying close attention to the potential of these advances technologies, something which is reflected in the multiple large scale Horizon 2020 funded EU projects had many successful outcomes to date with regard to advanced liver models for NBM toxicity screening [215-221]. In the US, the Wyss Institute is at the forefront of material and device bioprinting with their SWIFT technology, which is anticipated to be a silver bullet for resolving organ shortages worldwide [230]. Utilising 'organ-on-chip' technology, the Wyss Institute have also successfully reproduced many complex organ-level responses in no less than ten different organ-on-chip models [231], and have also developed a device to link them, in a 'body-on-a-chip' model $[232,233]$. Many of their innovations have also been commercially translated, with financial support obtained from many different government agencies, and they also work in partnership with various major pharmaceutical companies [214]. Further afield, countries like Brazil, Japan and China have also made great progress with the development of advanced hepatic models and 3D bioprinting technology [222-225]. The scientific culture within the biomedical field has certainly shifted in recent years, and animal experimentation and 2D in vitro pre-clinical assessment is not as immune to controversy and criticism as they once were. This shift is associated with the fact that, in recent years 3D models have been shown to be vastly more beneficial than conventional pre-clinical assessment methods 
in NBMs screening, with the intrinsic limitations of conventional in vitro and in vivo methods as the primary motivators for the development and validation of these new emerging 3D technologies. These physiologically relevant and advanced in vitro systems have the capacity to increase relevance of routine cell cultures while overcoming the interspecies differences characteristic of in vivo studies, thus becoming valuable tools for understanding the mechanisms of both drug and NBM toxicity. The ability to assess the fate and toxicity of NBMs on the liver in a human-relevant manner during their pre-clinical assessment is a huge advantage. Despite all of these advances however many roadblocks still exist, and even though there is a strong need for the availability of new, more predictive and ethical methodologies for NBM preclinical assessment, it may be many years until researchers, stakeholders, funding agencies and governments fully accept this change and make critical moves towards implementing these new methodologies. Thus, future efforts of the biomedical scientific community should aim at validating existing advanced methodologies and obtain regulatory approval ((e.g., OECD certification, EMA/FDA acceptance), an official recognition of the predictive value of the 3D advanced method proposed that will allow its widespread implementation across academia as well as industry.

\section{Declarations}

Ethical Statement

Ethics approval

$\mathrm{N} / \mathrm{A}$

Consent for publication

Consent has been asked where appropriate

\section{Availability of data and materials}

$\mathrm{N} / \mathrm{A}$

\section{Competing Interests}

Author $A, B$ and $C$ declare they have no competing financial interests.

\section{Funding}

This work was partially supported by the European Union H2020 framework programme under the REFINE project (Grant agreement ID: 761104).

\section{Authors' contribution}

All authors contributed equally to the drafting and finalization of this review.

\section{Acknowledgements}


The authors would like to thank all partners within REFINE and the LBCAM members, TCD

\section{Ethical approval}

N/A

\section{References}

1. Marques, M.R.C., et al., Nanomedicines - Tiny particles and big challenges. Adv Drug Deliv Rev, 2019. 151-152: p. 23-43.

2. Nanomedicine and the COVID-19 vaccines. Nature Nanotechnology, 2020. 15(12): p. 963-963.

3. van der Meel, R., T. Lammers, and W.E. Hennink, Cancer nanomedicines: oversold or underappreciated? Expert Opin Drug Deliv, 2017. 14(1): p. 1-5.

4. Soares, S., et al., Nanomedicine: Principles, Properties, and Regulatory Issues. Frontiers in Chemistry, 2018. 6(360).

5. Wolfram, J., et al., Safety of Nanoparticles in Medicine. Curr Drug Targets, 2015. 16(14): p. 167181.

6. Lee, W.M., Drug-induced hepatotoxicity. N Engl J Med, 2003. 349(5): p. 474-85.

7. Wu, T. and M. Tang, Review of the effects of manufactured nanoparticles on mammalian target organs. J Appl Toxicol, 2018. 38(1): p. 25-40.

8. Longmire, M., P.L. Choyke, and H. Kobayashi, Clearance properties of nano-sized particles and molecules as imaging agents: considerations and caveats. Nanomedicine (Lond), 2008. 3(5): p. 703-17.

9. Zhao, L. and B. Zhang, Doxorubicin induces cardiotoxicity through upregulation of death receptors mediated apoptosis in cardiomyocytes. Scientific Reports, 2017. 7(1): p. 44735.

10. Gabizon, A., et al., Clinical studies of liposome-encapsulated doxorubicin. Acta Oncol, 1994. 33(7): p. 779-86.

11. Hengge, U.R., et al., Fatal hepatic failure with liposomal doxorubicin. Lancet, 1993. 341(8841): p. 383-4.

12. Siegrist, S., et al., Preclinical hazard evaluation strategy for nanomedicines. Nanotoxicology, 2019. 13(1): p. 73-99.

13. Etheridge, M.L., et al., The big picture on nanomedicine: the state of investigational and approved nanomedicine products. Nanomedicine, 2013. 9(1): p. 1-14. 
14. Pelaz, B., et al., Diverse Applications of Nanomedicine. ACS Nano, 2017. 11(3): p. 2313-2381.

15. Collins SD, Y.G., Tu T, et al., In Vitro Models of the Liver: Disease Modeling, Drug Discovery and Clinical Applications., in Hepatocellular Carcinoma, T.-P. JEE, Editor. 2009.

16. Fröhlich, E., Comparison of conventional and advanced in vitro models in the toxicity testing of nanoparticles. Artif Cells Nanomed Biotechnol, 2018. 46(sup2): p. 1091-1107.

17. Kumar, V., N. Sharma, and S.S. Maitra, In vitro and in vivo toxicity assessment of nanoparticles. International Nano Letters, 2017. 7(4): p. 243-256.

18. Jensen, C. and Y. Teng, Is It Time to Start Transitioning From 2D to 3D Cell Culture? Frontiers in Molecular Biosciences, 2020. 7(33).

19. Collins, S.D., et al., In Vitro Models of the Liver: Disease Modeling, Drug Discovery and Clinical Applications, in Hepatocellular Carcinoma, J.E.E. Tirnitz-Parker, Editor. 2019, Codon Publications Brisbane (AU). p. 48-60.

20. Zhou, Y., J.X. Shen, and V.M. Lauschke, Comprehensive Evaluation of Organotypic and Microphysiological Liver Models for Prediction of Drug-Induced Liver Injury. 2019. 10(1093).

21. Soldatow, V.Y., et al., In vitro models for liver toxicity testing. Toxicol Res (Camb), 2013. 2(1): p. 23-39.

22. Vigue, J., Asklepios Atlas of Human Anatomy. 2014.

23. Portmann, B.C., Chapter 1 - Development and Anatomy of the Normal Liver, in Comprehensive Clinical Hepatology (Second Edition), B.R. Bacon, et al., Editors. 2006, Mosby: Edinburgh. p. 1-15.

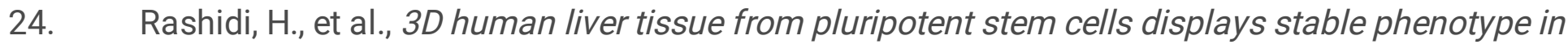
vitro and supports compromised liver function in vivo. Arch Toxicol, 2018. 92(10): p. 3117-3129.

25. LeCluyse, E.L., K.L. Audus, and J.H. Hochman, Formation of extensive canalicular networks by rat hepatocytes cultured in collagen-sandwich configuration. Am J Physiol, 1994. 266(6 Pt 1): p. C176474.

26. Choi, J.M., et al., HepG2 cells as an in vitro model for evaluation of cytochrome P450 induction by xenobiotics. Arch Pharm Res, 2015. 38(5): p. 691-704.

27. Elizondo, G. and I.M. Medina-Diaz, Induction of CYP3A4 by 1alpha,25-dyhydroxyvitamin D3 in HepG2 cells. Life Sci, 2003. 73(2): p. 141-9.

28. Liu, M.C., et al., Tyrosine sulfation of proteins from the human hepatoma cell line HepG2. Proc Natl Acad Sci U S A, 1985. 82(21): p. 7160-4. 
29. Vermeir, M., et al., Cell-based models to study hepatic drug metabolism and enzyme induction in humans. Expert Opin Drug Metab Toxicol, 2005. 1(1): p. 75-90.

30. Kermanizadeh, A., et al., An in vitro liver model - assessing oxidative stress and genotoxicity following exposure of hepatocytes to a panel of engineered nanomaterials. Particle and Fibre Toxicology, 2012. 9(1): p. 28.

31. Bandele, O.J., et al., In vitro toxicity screening of chemical mixtures using HepG2/C3A cells. Food Chem Toxicol, 2012. 50(5): p. 1653-9.

32. Bale, S.S., et al., In vitro platforms for evaluating liver toxicity. Exp Biol Med (Maywood), 2014. 239(9): p. 1180-1191.

33. Nibourg, G.A., et al., Proliferative human cell sources applied as biocomponent in bioartificial livers: a review. Expert Opin Biol Ther, 2012. 12(7): p. 905-21.

34. Guillouzo, A., et al., The human hepatoma HepaRG cells: a highly differentiated model for studies of liver metabolism and toxicity of xenobiotics. Chem Biol Interact, 2007. 168(1): p. 66-73.

35. Donato, M.T., et al., Cell lines: a tool for in vitro drug metabolism studies. Curr Drug Metab, 2008. 9(1): p. 1-11.

36. Gerets, H.H., et al., Characterization of primary human hepatocytes, HepG2 cells, and HepaRG cells at the mRNA level and CYP activity in response to inducers and their predictivity for the detection of human hepatotoxins. Cell Biol Toxicol, 2012. 28(2): p. 69-87.

37. O'Brien, P.J., et al., High concordance of drug-induced human hepatotoxicity with in vitro cytotoxicity measured in a novel cell-based model using high content screening. Arch Toxicol, 2006. 80(9): p. 580-604.

38. Zeilinger, K., et al., Cell sources for in vitro human liver cell culture models. Exp Biol Med (Maywood), 2016. 241(15): p. 1684-98.

39. Huang, J.R., et al., Liposomal Irinotecan for Treatment of Colorectal Cancer in a Preclinical Model. Cancers (Basel), 2019. 11(3).

40. Zhou, X., et al., Lactosylated liposomes for targeted delivery of doxorubicin to hepatocellular carcinoma. Int J Nanomedicine, 2012. 7: p. 5465-74.

41. Wang, P., et al., Evaluating cellular uptake of gold nanoparticles in HL-7702 and HepG2 cells for plasmonic photothermal therapy. Nanomedicine, 2018. 13(18): p. 2245-2259.

42. Rathinaraj, P., et al., Targeting and molecular imaging of HepG2 cells using surfacefunctionalized gold nanoparticles. Journal of Nanoparticle Research, 2015. 17(7): p. 311. 
43. Ashokkumar, T., et al., Apoptosis in liver cancer (HepG2) cells induced by functionalized gold nanoparticles. Colloids Surf B Biointerfaces, 2014. 123: p. 549-56.

44. Namvar, F., et al., Cytotoxic effect of magnetic iron oxide nanoparticles synthesized via seaweed aqueous extract. Int J Nanomedicine, 2014. 9: p. 2479-88.

45. Seo, D.Y., et al., Investigation of the genetic toxicity by dextran-coated superparamagnetic iron oxide nanoparticles (SPION) in HepG2 cells using the comet assay and cytokinesis-block micronucleus assay. Toxicology and Environmental Health Sciences, 2017. 9(1): p. 23-29.

46. Sulheim, E., et al., Cytotoxicity of Poly(Alkyl Cyanoacrylate) Nanoparticles. Int J Mol Sci, 2017. 18(11).

47. Sulheim, E., et al., Cellular uptake and intracellular degradation of poly(alkyl cyanoacrylate) nanoparticles. Journal of Nanobiotechnology, 2016. 14(1): p. 1.

48. Duan, J., et al., Cationic Polybutyl Cyanoacrylate Nanoparticles for DNA Delivery. Journal of Biomedicine and Biotechnology, 2009. 2009: p. 149254.

49. Ramboer, E., et al., Immortalized Human Hepatic Cell Lines for In Vitro Testing and Research Purposes. Methods Mol Biol, 2015. 1250: p. 53-76.

50. Prozialeck, W.C., et al., Epithelial barrier characteristics and expression of cell adhesion molecules in proximal tubule-derived cell lines commonly used for in vitro toxicity studies. Toxicol In Vitro, 2006. 20(6): p. 942-53.

51. Chamberlain, L.M., et al., Phenotypic non-equivalence of murine (monocyte-) macrophage cells in biomaterial and inflammatory models. J Biomed Mater Res A, 2009. 88(4): p. 858-71.

52. Milyavsky, M., et al., Prolonged culture of telomerase-immortalized human fibroblasts leads to a premalignant phenotype. Cancer Res, 2003. 63(21): p. 7147-57.

53. Ramboer, E., et al., Immortalized Human Hepatic Cell Lines for In Vitro Testing and Research Purposes. Methods in molecular biology (Clifton, N.J.), 2015. 1250: p. 53-76.

54. Soars, M.G., et al., The pivotal role of hepatocytes in drug discovery. Chem Biol Interact, 2007. 168(1): p. 2-15.

55. Zhou, Z., M.J. Xu, and B. Gao, Hepatocytes: a key cell type for innate immunity. Cell Mol Immunol, 2016. 13(3): p. 301-15.

56. Ponsoda, X., et al., Drug biotransformation by human hepatocytes. In vitro/in vivo metabolism by cells from the same donor. J Hepatol, 2001. 34(1): p. 19-25. 
57. Gomez-Lechon, M.J., et al., Human hepatocytes as a tool for studying toxicity and drug metabolism. Curr Drug Metab, 2003. 4(4): p. 292-312.

58. Gomez-Lechon, M.J., et al., Human hepatocytes in primary culture: the choice to investigate drug metabolism in man. Curr Drug Metab, 2004. 5(5): p. 443-62.

59. Knobeloch, D., et al., Human hepatocytes: isolation, culture, and quality procedures. Methods Mol Biol, 2012. 806: p. 99-120.

60. Rodriguez-Antona, C., et al., Cytochrome P450 expression in human hepatocytes and hepatoma cell lines: molecular mechanisms that determine lower expression in cultured cells. Xenobiotica, 2002. 32(6): p. 505-20.

61. Ranga, A., N. Gjorevski, and M.P. Lutolf, Drug discovery through stem cell-based organoid models. Adv Drug Deliv Rev, 2014. 69-70: p. 19-28.

62. Hartung, T. and G. Daston, Are in vitro tests suitable for regulatory use? Toxicol Sci, 2009. 111(2): p. 233-7.

63. Abdel-Misih, S.R. and M. Bloomston, Liver anatomy. Surg Clin North Am, 2010. 90(4): p. 643-53. 64. Granitzny, A., et al., Evaluation of a human in vitro hepatocyte-NPC co-culture model for the prediction of idiosyncratic drug-induced liver injury: A pilot study. Toxicol Rep, 2017. 4: p. 89-103.

65. Bale, S.S., et al., Isolation and co-culture of rat parenchymal and non-parenchymal liver cells to evaluate cellular interactions and response. Sci Rep, 2016. 6: p. 25329.

66. Ha, S.-W., et al., Chapter 4 - Applications of silica-based nanomaterials in dental and skeletal biology, in Nanobiomaterials in Clinical Dentistry (Second Edition), K. Subramani and W. Ahmed, Editors. 2019, Elsevier. p. 77-112.

67. Godoy, P., et al., Recent advances in 2D and 3D in vitro systems using primary hepatocytes, alternative hepatocyte sources and non-parenchymal liver cells and their use in investigating mechanisms of hepatotoxicity, cell signaling and ADME. Arch Toxicol, 2013. 87(8): p. 1315-530.

68. Bale, S.S., et al., Long-term coculture strategies for primary hepatocytes and liver sinusoidal endothelial cells. Tissue Eng Part C Methods, 2015. 21(4): p. 413-22.

69. Ohno, M., et al., Induction of drug-metabolizing enzymes by phenobarbital in layered co-culture of a human liver cell line and endothelial cells. Biol Pharm Bull, 2009. 32(5): p. 813-7.

70. Adams, D.H., et al., Mechanisms of immune-mediated liver injury. Toxicol Sci, 2010. 115(2): p. $307-21$. 
71. West, M.A., et al., Further characterization of Kupffer cell/macrophage-mediated alterations in hepatocyte protein synthesis. Surgery, 1986. 100(2): p. 416-423.

72. Kegel, V., et al., Subtoxic Concentrations of Hepatotoxic Drugs Lead to Kupffer Cell Activation in a Human In Vitro Liver Model: An Approach to Study DILI. Mediators Inflamm, 2015. 2015: p. 640631.

73. Yagi, K., et al., Stimulation of liver functions in hierarchical co-culture of bone marrow cells and hepatocytes. Cytotechnology, 1998. 26(1): p. 5-12.

74. Ha, S.-W., et al., Applications of silica-based nanomaterials in dental and skeletal biology, in Nanobiomaterials in Clinical Dentistry, K. Subramani and W. Ahmed, Editors. 2019, Elsevier. p. 77-112.

75. Edling, Y., et al., Increased sensitivity for troglitazone-induced cytotoxicity using a human in vitro co-culture model. Toxicol In Vitro, 2009. 23(7): p. 1387-95.

76. Esch, M.B., et al., Body-on-a-chip simulation with gastrointestinal tract and liver tissues suggests that ingested nanoparticles have the potential to cause liver injury. Lab Chip, 2014. 14(16): p. 3081-92.

77. Duval, K., et al., Modeling Physiological Events in 2D vs. 3D Cell Culture. Physiology (Bethesda), 2017. 32(4): p. 266-277.

78. Olsavsky Goyak, K.M., E.M. Laurenzana, and C.J. Omiecinski, Hepatocyte differentiation. Methods Mol Biol, 2010. 640: p. 115-38.

79. Kapalczynska, M., et al., 2D and 3D cell cultures - a comparison of different types of cancer cell cultures. Arch Med Sci, 2018. 14(4): p. 910-919.

80. Gissen, P. and I.M. Arias, Structural and functional hepatocyte polarity and liver disease. J Hepatol, 2015. 63(4): p. 1023-37.

81. Bacon. B. R., O.G.J.G., Di Bisceglie. A. M., Lake. J. R, Comprehensive Clinical Hepatology

82. Gomez-Lechon, M.J., et al., Long-term expression of differentiated functions in hepatocytes cultured in three-dimensional collagen matrix. J Cell Physiol, 1998. 177(4): p. 553-62.

83. Wells, R.G., The role of matrix stiffness in regulating cell behavior. Hepatology, 2008. 47(4): p. 1394-400.

84. Bell, C.C., et al., Comparison of Hepatic 2D Sandwich Cultures and 3D Spheroids for Long-term Toxicity Applications: A Multicenter Study. Toxicological sciences : an official journal of the Society of Toxicology, 2018. 162(2): p. 655-666. 
85. West, M.A., et al., Further characterization of Kupffer cell/macrophage-mediated alterations in hepatocyte protein synthesis. Surgery, 1986. 100(2): p. 416-23.

86. Mitragotri, S., et al., Drug Delivery Research for the Future: Expanding the Nano Horizons and Beyond. Journal of Controlled Release, 2017. 246: p. 183-184.

87. Yue, F., et al., A comparative encyclopedia of DNA elements in the mouse genome. Nature, 2014. 515(7527): p. 355-64.

88. Le Magnen, C., A. Dutta, and C. Abate-Shen, Optimizing mouse models for precision cancer prevention. Nat Rev Cancer, 2016. 16(3): p. 187-96.

89. Begley, C.G. and L.M. Ellis, Drug development: Raise standards for preclinical cancer research. Nature, 2012. 483(7391): p. 531-3.

90. Cook, N., D.I. Jodrell, and D.A. Tuveson, Predictive in vivo animal models and translation to clinical trials. Drug Discov Today, 2012. 17(5-6): p. 253-60.

91. Schachtschneider, K.M., et al., A validated, transitional and translational porcine model of hepatocellular carcinoma. Oncotarget, 2017. 8(38): p. 63620-63634.

92. Andrasina, T., et al., The Accumulation and Effects of Liposomal Doxorubicin in Tissues Treated by Radiofrequency Ablation and Irreversible Electroporation in Liver: In Vivo Experimental Study on Porcine Models. Cardiovasc Intervent Radiol, 2019. 42(5): p. 751-762.

93. Sieber, S., et al., Zebrafish as a preclinical in vivo screening model for nanomedicines. Advanced Drug Delivery Reviews, 2019. 151-152: p. 152-168.

94. Rennekamp, A.J. and R.T. Peterson, 15 years of zebrafish chemical screening. Curr Opin Chem Biol, 2015. 24: p. 58-70.

95. Peterson, R.T., et al., Small molecule developmental screens reveal the logic and timing of vertebrate development. Proc Natl Acad Sci U S A, 2000. 97(24): p. 12965-9.

96. Sieber, S., et al., Zebrafish as an early stage screening tool to study the systemic circulation of nanoparticulate drug delivery systems in vivo. J Control Release, 2017. 264: p. 180-191.

97. EC, Directive 2010/63/EU of the European Parliament and of the Council of 22 September 2020 on the Protection of Animals Used for Scientific Purposes. . 2010.

98. Goldsmith, P., Zebrafish as a pharmacological tool: the how, why and when. Current Opinion in Pharmacology, 2004. 4(5): p. 504-512.

99. Menke, A.L., et al., Normal Anatomy and Histology of the Adult Zebrafish. 2011. 39(5): p. 759775. 
100. Jung, H.M., et al., Development of the larval lymphatic system in zebrafish. Development, 2017. 144(11): p. 2070-2081.

101. Chen, A.T. and L.I. Zon, Zebrafish blood stem cells. J Cell Biochem, 2009. 108(1): p. 35-42.

102. Trede, N.S., et al., The use of zebrafish to understand immunity. Immunity, 2004. 20(4): p. 36779.

103. Isogai, S., M. Horiguchi, and B.M. Weinstein, The vascular anatomy of the developing zebrafish: an atlas of embryonic and early larval development. Dev Biol, 2001. 230(2): p. 278-301.

104. Chu, J. and K.C. Sadler, New school in liver development: lessons from zebrafish. Hepatology (Baltimore, Md.), 2009. 50(5): p. 1656-1663.

105. Howe, K., et al., The zebrafish reference genome sequence and its relationship to the human genome. Nature, 2013. 496(7446): p. 498-503.

106. Vibe, C.B., et al., Thioridazine in PLGA nanoparticles reduces toxicity and improves rifampicin therapy against mycobacterial infection in zebrafish. Nanotoxicology, 2016. 10(6): p. 680-688.

107. Peng, K., et al., Cyclodextrin/dextran based drug carriers for a controlled release of hydrophobic drugs in zebrafish embryos. Soft Matter, 2010. 6(16): p. 3778-3783.

108. Yan, H., et al., Functional Mesoporous Silica Nanoparticles for Photothermal-Controlled Drug Delivery In Vivo. 2012. 51(33): p. 8373-8377.

109. Fomchenko, E.I. and E.C. Holland, Mouse models of brain tumors and their applications in preclinical trials. Clin Cancer Res, 2006. 12(18): p. 5288-97.

110. Huff, J., M.F. Jacobson, and D.L. Davis, The limits of two-year bioassay exposure regimens for identifying chemical carcinogens. Environ Health Perspect, 2008. 116(11): p. 1439-42.

111. Van Norman, G.A., Limitations of Animal Studies for Predicting Toxicity in Clinical Trials: Is it Time to Rethink Our Current Approach? JACC Basic Transl Sci, 2019. 4(7): p. 845-854.

112. Van Norman, G.A., Drugs, Devices, and the FDA: Part 1: An Overview of Approval Processes for Drugs. JACC: Basic to Translational Science, 2016. 1(3): p. 170-179.

113. Shanks, N., R. Greek, and J. Greek, Are animal models predictive for humans? Philosophy, ethics, and humanities in medicine: PEHM, 2009. 4: p. 2-2.

114. Greek, R. and A. Menache, Systematic reviews of animal models: methodology versus epistemology. International journal of medical sciences, 2013. 10(3): p. 206-221. 
115. Hackam, D.G. and D.A. Redelmeier, Translation of research evidence from animals to humans. JAMA, 2006. 296(14): p. 1731-2.

116. Perel, P., et al., Comparison of treatment effects between animal experiments and clinical trials: systematic review. BMJ, 2007. 334(7586): p. 197.

117. Olson, H., et al., Concordance of the toxicity of pharmaceuticals in humans and in animals. Regul Toxicol Pharmacol, 2000. 32(1): p. 56-67.

118. Pound, P., et al., Where is the evidence that animal research benefits humans? BMJ, 2004. 328(7438): p. 514-7.

119. Bracken, M.B., Why animal studies are often poor predictors of human reactions to exposure. J R Soc Med, 2009. 102(3): p. 120-2.

120. Xu, J.J., D. Diaz, and P.J. O'Brien, Applications of cytotoxicity assays and pre-lethal mechanistic assays for assessment of human hepatotoxicity potential. Chem Biol Interact, 2004. 150(1): p. 115-28.

121. McKenzie, R., et al., Hepatic failure and lactic acidosis due to fialuridine (FIAU), an investigational nucleoside analogue for chronic hepatitis B. N Engl J Med, 1995. 333(17): p. 1099-105.

122. Institute of Medicine Committee to Review the Fialuridine Clinical, T., in Review of the Fialuridine (FIAU) Clinical Trials, F.J. Manning and M. Swartz, Editors. 1995, National Academies Press (US): Washington (DC).

123. Attarwala, H., TGN1412: From Discovery to Disaster. J Young Pharm, 2010. 2(3): p. 332-6.

124. Xu, D., et al., Fialuridine induces acute liver failure in chimeric TK-NOG mice: a model for detecting hepatic drug toxicity prior to human testing. PLoS Med, 2014. 11(4): p. e1001628.

125. Babai, S., L. Auclert, and H. Le-Louet, Safety data and withdrawal of hepatotoxic drugs. Therapie, 2018.

126. Villano, J.L., D. Mehta, and L. Radhakrishnan, Abraxane induced life-threatening toxicities with metastatic breast cancer and hepatic insufficiency. Invest New Drugs, 2006. 24(5): p. 455-6.

127. Socinski, M., Update on nanoparticle albumin-bound paclitaxel. Clin Adv Hematol Oncol, 2006. 4(10): p. 745-6.

128. Bracken, M.B., Why animal studies are often poor predictors of human reactions to exposure. Journal of the Royal Society of Medicine, 2009. 102(3): p. 120-122.

129. Administration, U.S.F.a.D., Investigational New Drug (IND) Application. U.S. Food and Drug Administration. 2017. 
130. Zhang, D., et al., Preclinical experimental models of drug metabolism and disposition in drug discovery and development. Acta Pharmaceutica Sinica B, 2012. 2(6): p. 549-561.

131. Akhtar, A., The flaws and human harms of animal experimentation. Camb Q Healthc Ethics, 2015. 24(4): p. 407-19.

132. Di Cristo, L., et al., Towards the Identification of an In Vitro Tool for Assessing the Biological Behavior of Aerosol Supplied Nanomaterials. Int J Environ Res Public Health, 2018. 15(4).

133. Bregoli, L., et al., Nanomedicine applied to translational oncology: A future perspective on cancer treatment. Nanomedicine, 2016. 12(1): p. 81-103.

134. Movia, D., et al., A safe-by-design approach to the development of gold nanoboxes as carriers for internalization into cancer cells. Biomaterials, 2014. 35(9): p. 2543-2557.

135. Movia, D., S. Bruni-Favier, and A. Prina-Mello, In vitro Alternatives to Acute Inhalation Toxicity Studies in Animal Models-A Perspective. Frontiers in bioengineering and biotechnology, 2020. 8: p. 549549.

136. Movia, D. and A. Prina-Mello, Preclinical Development of Orally Inhaled Drugs (OIDs)-Are Animal Models Predictive or Shall We Move Towards In Vitro Non-Animal Models? Animals (Basel), 2020. 10(8).

137. Prina-Mello, A., et al., Editorial: Use of 3D Models in Drug Development and Precision Medicine Advances and Outlook. Frontiers in Bioengineering and Biotechnology, 2021. 9(137).

138. Prina-Mello, D.M.a.A., Nanotoxicity in Cancer Research: Technical Protocols and Considerations for the Use of 3D Tumour Spheroids, Unraveling the Safety Profile of Nanoscale Particles and Materials, in From Biomedical to Environmental Applications. 2017: IntechOpen.

139. Alepee, N., et al., State-of-the-art of 3D cultures (organs-on-a-chip) in safety testing and pathophysiology. ALTEX, 2014. 31(4): p. 441-77.

140. Fleddermann, J., et al., Distribution of SiO2 nanoparticles in 3D liver microtissues. Int J Nanomedicine, 2019. 14: p. 1411-1431.

141. Ozkan, A., et al., In vitro vascularized liver and tumor tissue microenvironments on a chip for dynamic determination of nanoparticle transport and toxicity. Biotechnol Bioeng, 2019. 116(5): p. 12011219.

142. Otieno, M.A., J. Gan, and W. Proctor, Status and Future of 3D Cell Culture in Toxicity Testing, in Drug-Induced Liver Toxicity, M. Chen and Y. Will, Editors. 2018, Springer New York: New York, NY. p. 249261. 
143. Duval, K., et al., Modeling Physiological Events in 2D vs. 3D Cell Culture. Physiology (Bethesda, Md.), 2017. 32(4): p. 266-277.

144. Fang, Y. and R.M. Eglen, Three-Dimensional Cell Cultures in Drug Discovery and Development. SLAS Discov, 2017. 22(5): p. 456-472.

145. Deng, J., et al., Engineered Liver-on-a-Chip Platform to Mimic Liver Functions and Its Biomedical Applications: A Review. Micromachines (Basel), 2019. 10(10): p. 676.

146. Dunn, J.C., R.G. Tompkins, and M.L. Yarmush, Hepatocytes in collagen sandwich: evidence for transcriptional and translational regulation. J Cell Biol, 1992. 116(4): p. 1043-53.

147. Dunn, J.C., R.G. Tompkins, and M.L. Yarmush, Long-term in vitro function of adult hepatocytes in a collagen sandwich configuration. Biotechnol Prog, 1991. 7(3): p. 237-45.

148. Dunn, J.C., et al., Hepatocyte function and extracellular matrix geometry: long-term culture in a sandwich configuration. FASEB J, 1989. 3(2): p. 174-7.

149. Molina-Jimenez, F., et al., Matrigel-embedded 3D culture of Huh-7 cells as a hepatocyte-like polarized system to study hepatitis C virus cycle. Virology, 2012. 425(1): p. 31-9.

150. Swift, B., N.D. Pfeifer, and K.L.R. Brouwer, Sandwich-cultured hepatocytes: an in vitro model to evaluate hepatobiliary transporter-based drug interactions and hepatotoxicity. Drug metabolism reviews, 2010. 42(3): p. 446-471.

151. Liu, X., et al., Partial maintenance of taurocholate uptake by adult rat hepatocytes cultured in a collagen sandwich configuration. Pharm Res, 1998. 15(10): p. 1533-9.

152. Molina-Jimenez, F., et al., Matrigel-embedded 3D culture of Huh-7 cells as a hepatocyte-like polarized system to study hepatitis $C$ virus cycle. Virology, 2012. 425(1): p. 31-39.

153. Ruoss, M., et al., A Standardized Collagen-Based Scaffold Improves Human Hepatocyte Shipment and Allows Metabolic Studies over 10 Days. Bioengineering (Basel), 2018. 5(4): p. 86.

154. Lee, J., et al., In vitro toxicity testing of nanoparticles in 3D cell culture. Small, 2009. 5(10): p. 1213-21.

155. Haldar, S., D. Lahiri, and P. Roy, Chapter 5 - 3D Print Technology for Cell Culturing, in 3D Printing Technology in Nanomedicine, N. Ahmad, P. Gopinath, and R. Dutta, Editors. 2019, Elsevier. p. 83-114.

156. Chang, R., et al., Biofabrication of a three-dimensional liver micro-organ as an in vitro drug metabolism model. Biofabrication, 2010. 2(4): p. 045004.

157. Ma, X., et al., Deterministically patterned biomimetic human iPSC-derived hepatic model via rapid 3D bioprinting. Proc Natl Acad Sci U S A, 2016. 113(8): p. 2206-11. 
158. Nguyen, A.H., et al., MMP-mediated mesenchymal morphogenesis of pluripotent stem cell aggregates stimulated by gelatin methacrylate microparticle incorporation. Biomaterials, 2016. 76: p. 6675 .

159. Nguyen, D.G., et al., Bioprinted 3D Primary Liver Tissues Allow Assessment of Organ-Level Response to Clinical Drug Induced Toxicity In Vitro. PLoS One, 2016. 11(7): p. e0158674.

160. Pampaloni, F. and E. Stelzer, Three-dimensional cell cultures in toxicology. Biotechnol Genet Eng Rev, 2010. 26: p. 117-38.

161. Wong, S.F., et al., Concave microwell based size-controllable hepatosphere as a threedimensional liver tissue model. Biomaterials, 2011. 32(32): p. 8087-96.

162. Friedrich, J., et al., Spheroid-based drug screen: considerations and practical approach. Nat Protoc, 2009. 4(3): p. 309-24.

163. Foty, R., A simple hanging drop cell culture protocol for generation of 3D spheroids. Journal of visualized experiments : JoVE, 2011(51): p. 2720.

164. Achilli, T.-M., J. Meyer, and J.R. Morgan, Advances in the formation, use and understanding of multi-cellular spheroids. Expert opinion on biological therapy, 2012. 12(10): p. 1347-1360.

165. Otsuka, H., et al., Micropatterned co-culture of hepatocyte spheroids layered on non-parenchymal cells to understand heterotypic cellular interactions. Science and technology of advanced materials, 2013. 14(6): p. 065003-065003.

166. Edmondson, R., et al., Three-dimensional cell culture systems and their applications in drug discovery and cell-based biosensors. Assay and drug development technologies, 2014. 12(4): p. 207-218.

167. Chang, T.T. and M. Hughes-Fulford, Monolayer and spheroid culture of human liver hepatocellular carcinoma cell line cells demonstrate distinct global gene expression patterns and functional phenotypes. Tissue Eng Part A, 2009. 15(3): p. 559-67.

168. Fey, S.J. and K. Wrzesinski, Determination of drug toxicity using 3D spheroids constructed from an immortal human hepatocyte cell line. Toxicol Sci, 2012. 127(2): p. 403-11.

169. Vorrink, S.U., et al., Prediction of Drug-Induced Hepatotoxicity Using Long-Term Stable Primary Hepatic 3D Spheroid Cultures in Chemically Defined Conditions. Toxicol Sci, 2018. 163(2): p. 655-665.

170. Messner, S., et al., Multi-cell type human liver microtissues for hepatotoxicity testing. Arch Toxicol, 2013. 87(1): p. 209-13.

171. Tostoes, R.M., et al., Human liver cell spheroids in extended perfusion bioreactor culture for repeated-dose drug testing. Hepatology, 2012. 55(4): p. 1227-36. 
172. Peshwa, M.V., et al., Mechanistics of formation and ultrastructural evaluation of hepatocyte spheroids. In Vitro Cell Dev Biol Anim, 1996. 32(4): p. 197-203.

173. Riccalton-Banks, L., et al., Long-term culture of functional liver tissue: three-dimensional coculture of primary hepatocytes and stellate cells. Tissue Eng, 2003. 9(3): p. 401-10.

174. Ramaiahgari, S.C., et al., $A 3 D$ in vitro model of differentiated HepG2 cell spheroids with improved liver-like properties for repeated dose high-throughput toxicity studies. Arch Toxicol, 2014. 88(5): p. 1083-95.

175. Elje, E., et al., Hepato(Geno)Toxicity Assessment of Nanoparticles in a HepG2 Liver Spheroid Model. Nanomaterials (Basel), 2020. 10(3).

176. Dubiak-Szepietowska, M., et al., Development of complex-shaped liver multicellular spheroids as a human-based model for nanoparticle toxicity assessment in vitro. Toxicol Appl Pharmacol, 2016. 294: p. 78-85.

177. Mikhail, A.S., S. Eetezadi, and C. Allen, Multicellular tumor spheroids for evaluation of cytotoxicity and tumor growth inhibitory effects of nanomedicines in vitro: a comparison of docetaxelloaded block copolymer micelles and Taxotere(R). PLoS One, 2013. 8(4): p. e62630.

178. Huch, M., et al., Long-term culture of genome-stable bipotent stem cells from adult human liver. Cell, 2015. 160(1-2): p. 299-312.

179. Prior, N., P. Inacio, and M. Huch, Liver organoids: from basic research to therapeutic applications. Gut, 2019. 68(12): p. 2228-2237.

180. Akbari, S., et al., Next-Generation Liver Medicine Using Organoid Models. Front Cell Dev Biol, 2019. 7: p. 345.

181. Palazzolo, S., et al., An Effective Multi-Stage Liposomal DNA Origami Nanosystem for In Vivo Cancer Therapy. Cancers (Basel), 2019. 11(12).

182. de Graaf, I.A., et al., Preparation and incubation of precision-cut liver and intestinal slices for application in drug metabolism and toxicity studies. Nat Protoc, 2010. 5(9): p. 1540-51.

183. Palma, E., E.J. Doornebal, and S. Chokshi, Precision-cut liver slices: a versatile tool to advance liver research. Hepatol Int, 2019. 13(1): p. 51-57.

184. Wu, X., et al., Precision-cut human liver slice cultures as an immunological platform. J Immunol Methods, 2018. 455: p. 71-79.

185. Palma, E., E.J. Doornebal, and S. Chokshi, Precision-cut liver slices: a versatile tool to advance liver research. Hepatology International, 2019. 13(1): p. 51-57. 
186. Olinga, P., et al., Rat liver slices as a tool to study LPS-induced inflammatory response in the liver. J Hepatol, 2001. 35(2): p. 187-94.

187. Dragoni, S., et al., Gold Nanoparticles Uptake and Cytotoxicity Assessed on Rat Liver PrecisionCut Slices. Toxicological Sciences, 2012. 128(1): p. 186-197.

188. Hui, A.Y. and S.L. Friedman, Molecular basis of hepatic fibrosis. Expert Rev Mol Med, 2003. 5(5): p. 1-23.

189. Bartucci, R., et al., Time-Resolved Quantification of Nanoparticle Uptake, Distribution, and Impact in Precision-Cut Liver Slices. Small, 2020. 16(21): p. 1906523.

190. van Midwoud, P.M., et al., A microfluidic approach for in vitro assessment of interorgan interactions in drug metabolism using intestinal and liver slices. Lab Chip, 2010. 10(20): p. 2778-86.

191. Vaira, V., et al., Preclinical model of organotypic culture for pharmacodynamic profiling of human tumors. Proceedings of the National Academy of Sciences, 2010. 107(18): p. 8352-8356.

192. Hattersley, S.M., J. Greenman, and S.J. Haswell, Study of ethanol induced toxicity in liver explants using microfluidic devices. Biomed Microdevices, 2011. 13(6): p. 1005-14.

193. Freeman, A.E. and R.M. Hoffman, In vivo-like growth of human tumors in vitro. Proc Natl Acad Sci U S A, 1986. 83(8): p. 2694-8.

194. Vaira, V., et al., Preclinical model of organotypic culture for pharmacodynamic profiling of human tumors. Proc Natl Acad Sci U S A, 2010. 107(18): p. 8352-6.

195. Piera, T., Organ Culture Model of Liver for the Study of Cancer Treatment for Hepatocellular Carcinoma. Cancer Research Journal, 2016. 4: p. 37.

196. Nath, S. and G.R. Devi, Three-dimensional culture systems in cancer research: Focus on tumor spheroid model. Pharmacol Ther, 2016. 163: p. 94-108.

197. Toh, Y.C., et al., A microfluidic 3D hepatocyte chip for drug toxicity testing. Lab Chip, 2009. 9(14): p. 2026-35.

198. Filippi, C., et al., Improvement of C3A cell metabolism for usage in bioartificial liver support systems. J Hepatol, 2004. 41(4): p. 599-605.

199. Gebhardt, R. and D. Mecke, Perifused monolayer cultures of rat hepatocytes as an improved in vitro system for studies on ureogenesis. Experimental Cell Research, 1979. 124(2): p. 349-359.

200. Bhatia, S.N. and D.E. Ingber, Microfluidic organs-on-chips. Nat Biotechnol, 2014. 32(8): p. 760-72. 
201. Gerlach, J.C., et al., Use of primary human liver cells originating from discarded grafts in a bioreactor for liver support therapy and the prospects of culturing adult liver stem cells in bioreactors: a morphologic study. Transplantation, 2003. 76(5): p. 781-6.

202. Bhise, N.S., et al., Organ-on-a-chip platforms for studying drug delivery systems. J Control Release, 2014. 190: p. 82-93.

203. Gebhardt, R., et al., New hepatocyte in vitro systems for drug metabolism: metabolic capacity and recommendations for application in basic research and drug development, standard operation procedures. Drug Metab Rev, 2003. 35(2-3): p. 145-213.

204. Li, L., et al., A microfluidic 3D hepatocyte chip for hepatotoxicity testing of nanoparticles. Nanomedicine (Lond), 2019. 14(16): p. 2209-2226.

205. Liu, Y., S. Wang, and Y. Wang, Patterned Fibers Embedded Microfluidic Chips Based on PLA and PDMS for Ag Nanoparticle Safety Testing. Polymers (Basel), 2016. 8(11).

206. Wikswo, J.P., The relevance and potential roles of microphysiological systems in biology and medicine. Experimental biology and medicine (Maywood, N.J.), 2014. 239(9): p. 1061-1072.

207. Ashammakhi, N., et al., Microphysiological Systems: Next Generation Systems for Assessing Toxicity and Therapeutic Effects of Nanomaterials. 2020. 4(1): p. 1900589.

208. Esch, M.B., et al., Body-on-a-chip simulation with gastrointestinal tract and liver tissues suggests that ingested nanoparticles have the potential to cause liver injury. Lab on a chip, 2014. 14(16): p. 30813092.

209. Zhang, Y.S., Y.-N. Zhang, and W. Zhang, Cancer-on-a-chip systems at the frontier of nanomedicine. Drug discovery today, 2017. 22(9): p. 1392-1399.

210. Lee, S.A., et al., Spheroid-based three-dimensional liver-on-a-chip to investigate hepatocytehepatic stellate cell interactions and flow effects. Lab Chip, 2013. 13(18): p. 3529-37.

211. Louhimies, S., Directive 86/609/EEC on the protection of animals used for experimental and other scientific purposes. Altern Lab Anim, 2002. 30 Suppl 2: p. 217-9.

212. Segovia-Miranda, F., et al., Three-dimensional spatially resolved geometrical and functional models of human liver tissue reveal new aspects of NAFLD progression. Nat Med, 2019. 25(12): p. 18851893.

213. Neff, E.P., Printing cures: Organovo advances with 3D-printed liver tissue. Lab Anim (NY), 2017. 46(3): p. 57. 
214. Tolikas, M., A. Antoniou, and D.E. Ingber, The Wyss institute: A new model for medical technology innovation and translation across the academic-industrial interface. Bioeng Transl Med, 2017. 2(3): p. 247-257.

215. Conway, G.E., et al., Adaptation of the in vitro micronucleus assay for genotoxicity testing using 3D liver models supporting longer-term exposure durations. Mutagenesis, 2020.

216. Au - Llewellyn, S.V., et al., Advanced 3D Liver Models for In vitro Genotoxicity Testing Following Long-Term Nanomaterial Exposure. JoVE, 2020(160): p. e61141.

217. Kermanizadeh, A., et al., The importance of inter-individual Kupffer cell variability in the governance of hepatic toxicity in a 3D primary human liver microtissue model. Scientific Reports, 2019. 9(1): p. 7295.

218. Barosova, H., et al., An In Vitro Lung System to Assess the Proinflammatory Hazard of Carbon Nanotube Aerosols. Int J Mol Sci, 2020. 21(15).

219. Au - Braakhuis, H.M., et al., An Air-liquid Interface Bronchial Epithelial Model for Realistic, Repeated Inhalation Exposure to Airborne Particles for Toxicity Testing. JoVE, 2020(159): p. e61210.

220. Au - Barosova, H., et al., Multicellular Human Alveolar Model Composed of Epithelial Cells and Primary Immune Cells for Hazard Assessment. JoVE, 2020(159): p. e61090.

221. Ude, V.C., et al., Using 3D gastrointestinal tract in vitro models with microfold cells and mucus secreting ability to assess the hazard of copper oxide nanomaterials. J Nanobiotechnology, 2019. 17(1): p. 70.

222. Goulart, E., et al., 3D bioprinting of liver spheroids derived from human induced pluripotent stem cells sustain liver function and viability in vitro. Biofabrication, 2019. 12(1): p. 015010.

223. Yang, $\mathrm{H}_{\text {., }}$ et al., Three-dimensional bioprinted hepatorganoids prolong survival of mice with liver failure. Gut, 2020: p. gutjnl-2019-319960.

224. Wang, L., et al., Automated quantitative assessment of three-dimensional bioprinted hydrogel scaffolds using optical coherence tomography. Biomed Opt Express, 2016. 7(3): p. 894-910.

225. Kizawa, H., et al., Scaffold-free 3D bio-printed human liver tissue stably maintains metabolic functions useful for drug discovery. Biochemistry and Biophysics Reports, 2017. 10: p. 186-191.

226. NCATS. https://ncats.nih.gov/pubs/features/3d-bioprinting. 2018; Available from: https://ncats.nih.gov/pubs/features/3d-bioprinting.

227. Livingston, C.A., K.M. Fabre, and D.A. Tagle, Facilitating the commercialization and use of organ platforms generated by the microphysiological systems (Tissue Chip) program through public-private 
partnerships. Computational and Structural Biotechnology Journal, 2016. 14: p. 207-210.

228. Kaiser, J. Seven years later, NIH center that aims to speed drugs to market faces challenges. 2019 [cited 2022 04/01]; Available from: https://www.sciencemag.org/news/2019/09/seven-years-laternih-center-aims-speed-drugs-market-faces-challenges.

229. Zhang, Y.S., et al., 3D Bioprinting for Tissue and Organ Fabrication. Ann Biomed Eng, 2017. 45(1): p. 148-163.

230. Brownell, L. A swifter way towards 3D-printed organs. 2019 [cited 2022 04/01]; Available from: https://wyss.harvard.edu/news/a-swifter-way-towards-3d-printed-organs/.

231. Huh, D., et al., Reconstituting organ-level lung functions on a chip. Science, 2010. 328(5986): p. 1662-8.

232. Herland, A., et al., Quantitative prediction of human pharmacokinetic responses to drugs via fluidically coupled vascularized organ chips. Nature Biomedical Engineering, 2020. 4(4): p. 421-436.

233. Novak, R., et al., Robotic fluidic coupling and interrogation of multiple vascularized organ chips. Nature Biomedical Engineering, 2020. 4(4): p. 407-420.

\section{Figures}
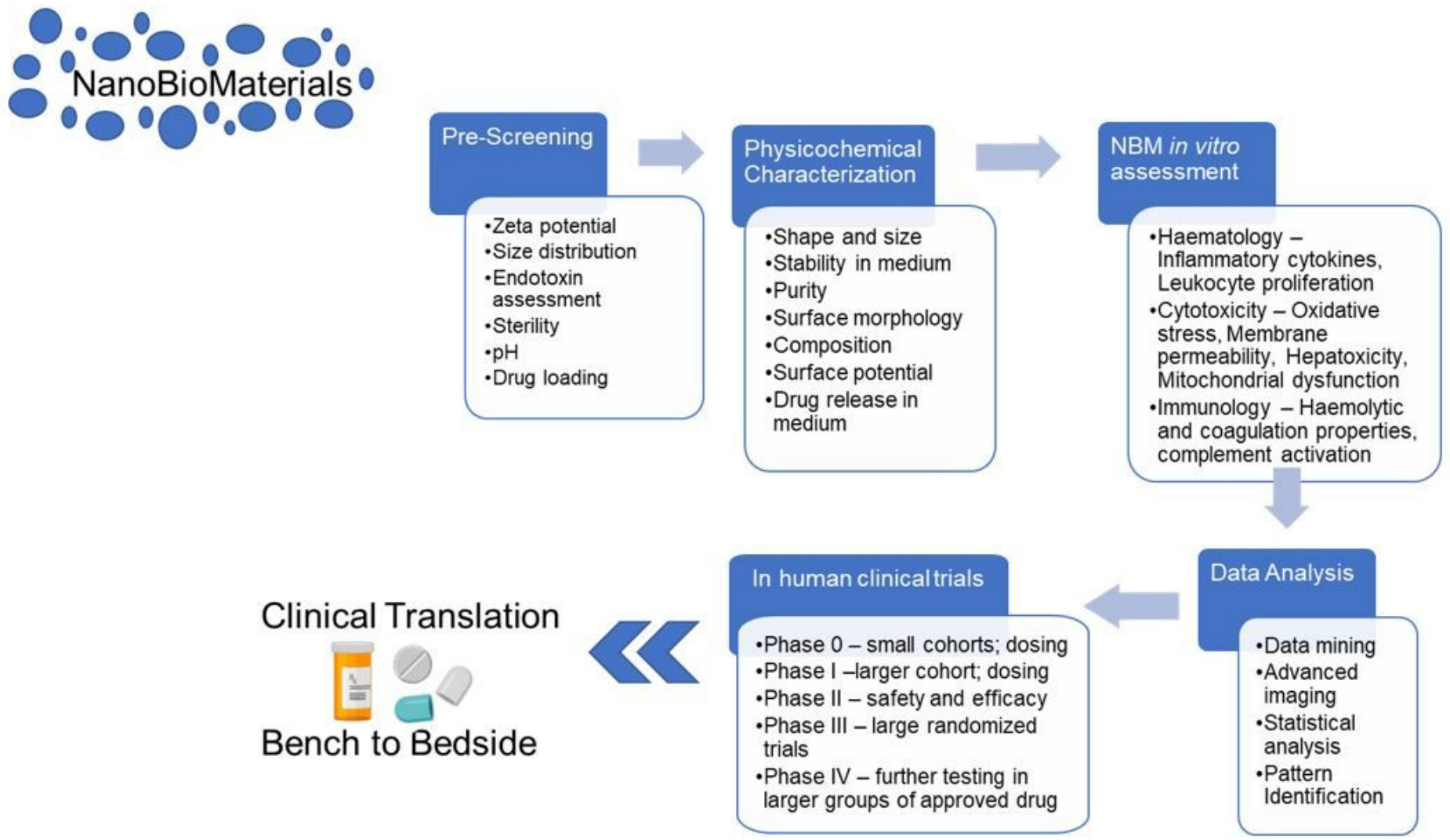
Figure 1

The pre-clinical assessment cascade for assessing NBM safety and efficacy. Following pre-screening and sterility assessment, a candidate NBM passes through physico-chemical characterization, in vitro and in vivo experiments, before being deemed safe to enter human clinical trials.

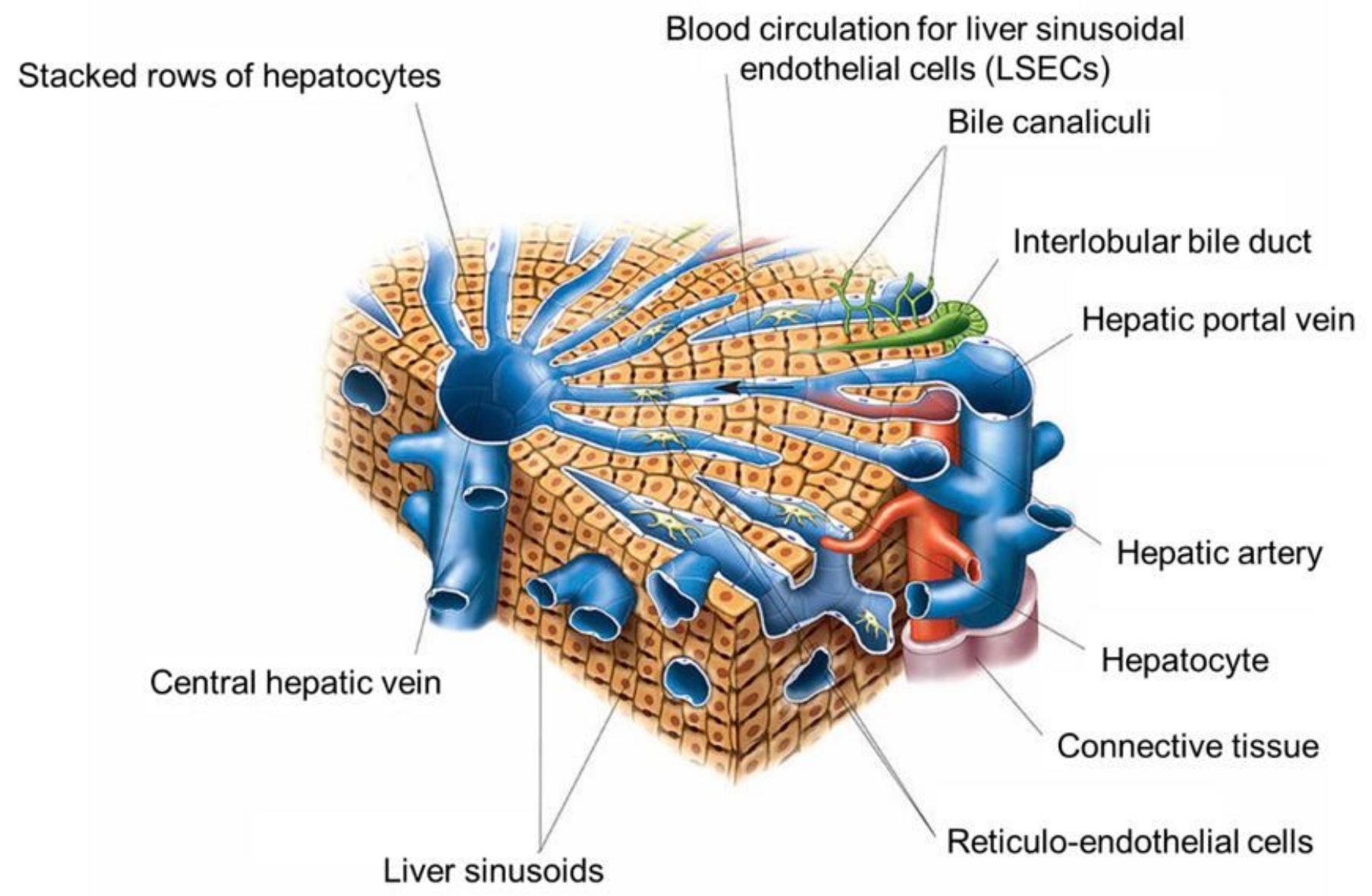

Figure 2

Structure of the Lobule of Liver. This illustration includes many key elements of liver phenotype and function that cannot be successfully replicated in 2D culture, including ordered stacking of hepatocytes, formation of bile canaliculi and blood circulation. Adapted from 'Asklepios Atlas of the Human Anatomy'[22]. 


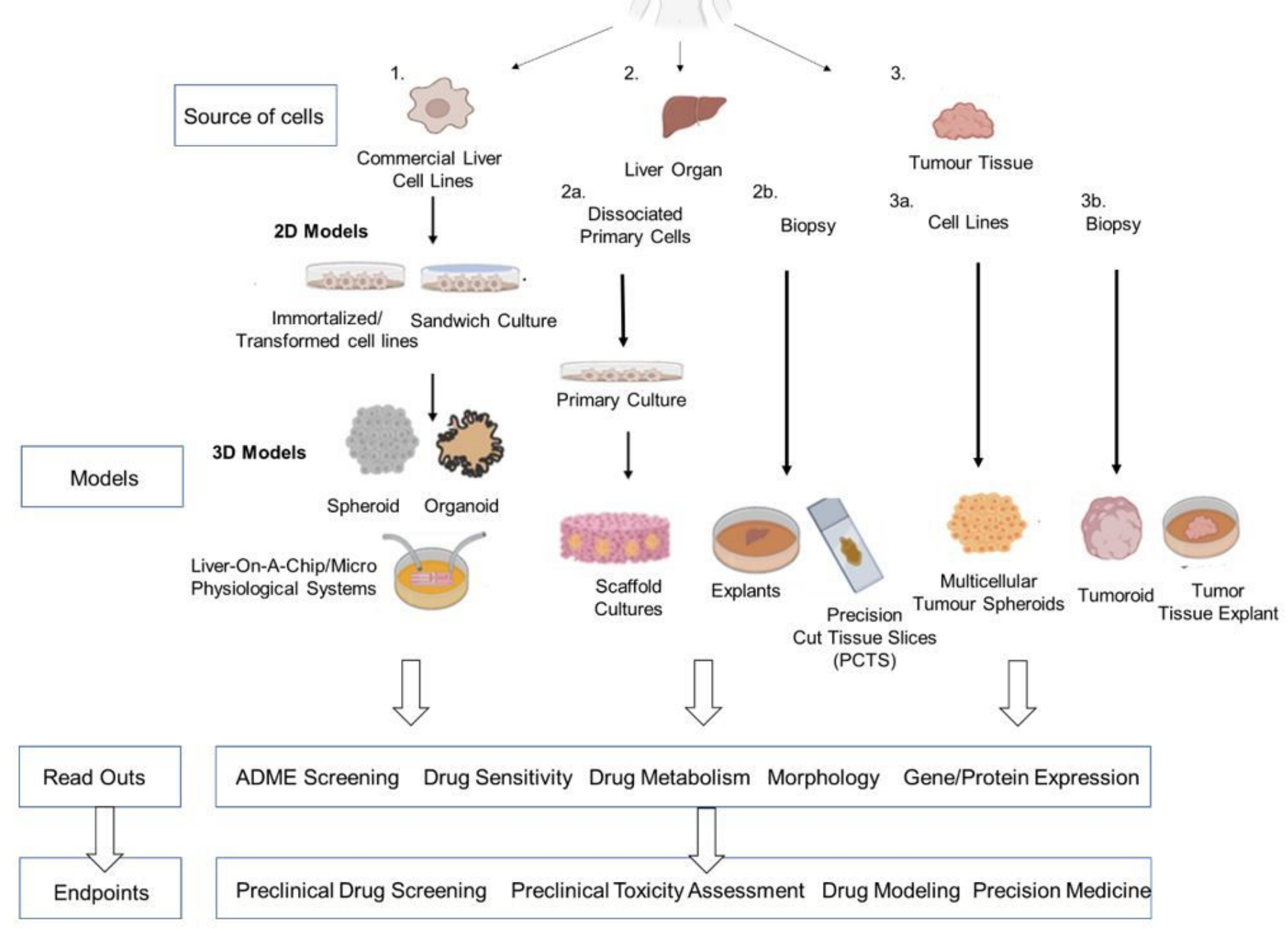

Figure 3

Overview of the various 2D and 3D in vitro models of the liver. Flow diagram illustrates the source of liver cell/tissue, their potential 2D and 3D models, their readouts, and associated endpoints. Adapted from Hepatocellular Carcinoma, Chapter 3: In Vitro Models of the Liver: Disease Modelling, Drug Discovery and Clinical Applications [15].

\section{Figure 4}

The most common methods for spheroid formation, cultivation, and growth. A variety of different techniques for 3D spheroid production exist, each varying in complexity and ease of use. Common techniques include (A) Static suspensions, or the liquid overlay technique, with spheroids formed from 
interruption of cell adhesion on non-adherent surfaces, (B) Hanging drops, undertaken using commercial systems like InSphero ${ }^{\mathrm{TM}}$ Gravity Plus ${ }^{\mathrm{TM}}$, or using upturned petri dishes, whereby cells are seeded in small drops in medium and spheroids form due to gravitational forces, (C) Rotary and spinning cultures, formed in vessels specifically designed to prevent cell adhesion, (D) Magnet assisted cultures or magnetic levitation, where cells are magnetised in culture, often by using nanoparticles, and pulled towards a magnetic on top of culture vessel, and (E) Ultra-low attachment (ULA) surfaces and plates. Each technique is based on the principle to force cells to self-aggregate and compact into spheroidal 3D microtissues.

\section{Supplementary Files}

This is a list of supplementary files associated with this preprint. Click to download.

- graphicsabstract.jpg 